\title{
Title: $\quad$ Spiking network optimized for noise robust word recognition approaches human-level performance and predicts auditory system hierarchy
}

\author{
Authors: $\quad$ Fatemeh Khatami $^{1}$ and Monty A. Escabi ${ }^{1,2,3}$ \\ Affiliation: Department of Biomedical Engineering ${ }^{1}$, Department of Electrical and \\ Computer Engineering ${ }^{2}$, and Department of Psychological Sciences ${ }^{3}$, \\ University of Connecticut, Storrs, CT 06109
}

\author{
Correspondence: $\quad$ Monty A. Escabí \\ Department of Electrical and Computer Engineering \\ 371 Fairfield Way, U4157 \\ Storrs, CT 06269 \\ escabi@engr.uconn.edu \\ Manuscript Info: 7 figures, 165 (abstract), 235 (introduction), 738 (discussion) \\ Conflict of Interest: None
}

Author Contribution: M.A.E. developed the auditory HSSN model. F.K. optimized and refined the model and analyzed the data. M.A.E. and F.K. contributed to manuscript preparation.

Acknowledgments: We thank Heather Read and Ian Stevenson for providing feedback on the manuscript and E.D. Young for providing auditory nerve data. Research reported in this publication was partly supported by the National Institute On Deafness And Other Communication Disorders of the National Institutes of Health under Award Number R01DC015138 and a grant from the University of Connecticut Research Foundation. The content is solely the responsibility of the authors and does not necessarily represent the official views of the National Institutes of Health.

Significance Statement: The brain's ability to recognize sounds in the presence of competing sounds or background noise is essential for everyday hearing tasks. How the brain accomplishes noise resiliency, however, is poorly understood. Using neural recording from the ascending auditory pathway and an auditory spiking network model trained for optimal sound recognition in noise we explore the computational strategies that enable noise robustness. Our results suggest that the hierarchical organization of the auditory pathway and the resulting nonlinear transformations may form a near optimal strategy that is essential for sound recognition in the presence of noise.

Keywords: auditory system, hearing, speech recognition, background noise, spectro-temporal, 


\section{Abstract}

The auditory neural code is resilient to acoustic variability and capable of recognizing

45 sounds amongst competing sound sources, yet, the transformations enabling noise robust abilities

46 are largely unknown. We report that a hierarchical spiking neural network (HSNN) trained to

47 maximize word recognition accuracy in noise and multiple talkers approaches human-level

48 performance. Intriguingly, comparisons with data from auditory nerve, midbrain, thalamus and

49 cortex reveals that the organization and nonlinear transformations of the optimal network predict

50 several properties of the ascending auditory pathway including a sequential loss of temporal

51 resolution, increasing sparseness and selectivity. The optimal organizational scheme is critical for

52 noise robustness since an identical network arranged to enable high information transfer does not

53 predict auditory pathway organization and has substantially poorer performance. Furthermore,

54 conventional linear and nonlinear receptive field-based models fail to achieve similar noise robust

55 performance. The findings suggest that the auditory pathway hierarchy and its sequential nonlinear

56 feature extraction computations may form a near optimal code capable of efficiently detecting

57 sounds in noise impoverished conditions.

58

59

60

61 facilitate robust coding of sounds ${ }^{1,2}$ it is presently unclear how the sequential organization of the

62 ascending auditory pathway and the resulting nonlinear transformations contribute to robust sound

63

64

65

\section{Introduction}

Being able to identify sounds in the presence of background noise is essential for everyday audition and vital for survival. Although several cortical mechanisms have been proposed to recognition.

Several hierarchical changes in spectral and temporal selectivity are consistently observed in the ascending auditory pathway of mammals. Temporal selectivity and resolution change 
66 dramatically over more than an order of magnitude, from a high-resolution representation in the

67 cochlea, where auditory nerve fibers synchronize to temporal features of up to $\sim 1000 \mathrm{~Hz}$, to

68 progressively slower (limited to $\sim 25 \mathrm{~Hz}$ ) and coarser resolution representation as observed in

69 auditory cortex ${ }^{3}$. Furthermore, although changes in spectral selectivity can be described across

70 different stages of the auditory pathway, and spectral resolution is somewhat coarser in central

71 levels, changes in frequency resolution are somewhat more homogeneous and less dramatic ${ }^{4-6}$. It

72 is plausible that such hierarchical transforms across auditory nuclei are essential for feature

73 extraction and ultimately high-level auditory tasks such as acoustic object recognition. Yet, it is

74 unclear whether these sequential transformations comprise an optimal computational strategy for

75 noise robust sound encoding. Here we report that the hierarchical organization of the auditory

76 pathway and its sequential nonlinear feature extraction transformations form a near-optimal

77 computation strategy for noise robust sound coding.

78

79 RESULTS

80 Task optimized hierarchical spiking neural network predicts auditory system organization

81 We developed a physiologically motivated hierarchical spiking neural network (HSNN)

82 and trained it on a behaviorally relevant word recognition task in the presence of background noise

83 and multiple talkers. Like the auditory pathway, the HSNN receives frequency-organized input

84 from a cochlear stage (Fig. 1a) and maintains its topographic (tonotopic) organization through a

85 network of frequency organized integrate-and-fire spiking neurons (Fig. 1b). For each sound, such

86 as the word "zero", the network produces a dynamic spatio-temporal pattern of spiking activity

87 (Fig. 1b, right) as observed for peripheral and central auditory structures ${ }^{7-9}$. Each neuron is highly 
10-13 that project across six network layers (Fig. 1b). Converging spikes from neurons in a given layer (Fig 1d) are weighted by frequency localized excitatory and inhibitory connectivity functions

91 and the resulting excitatory and inhibitory post-synaptic potentials are integrated by the recipient neuron (Fig. 1d and e, note the variable spike amplitudes). Output spike trains from each neuron are then weighted by connectivity function, providing the excitatory and inhibitory inputs to the next layer (Fig. 1e, f). The overall multi-neuron spiking output of the network (Fig. 1b, right) is then treated as a response feature vector and fed to a Bayesian classifier in order to identify the original sound delivered (Fig. 1c; see Methods). and temporal composition that are critical for word identification ${ }^{14,15}$, we first test how the spectrotemporal resolution and sensitivity of each network layer contribute to word recognition in the presence of noise and to identify the network organization of three key parameters that separately control the temporal and spectral resolution and the overall sensitivity of each network layer $(l=1 \ldots 6)$. The neuron time-constant $\left(\tau_{l}\right)$, controls the temporal dynamics of each neuron element in layer $l$ and the resulting temporal resolution of the output spiking patterns. The connectivity width $\left(\sigma_{l}\right)$ controls the convergence and divergence of synaptic connections between consecutive layers and therefore affects the spectral resolution of each layer. Since synaptic connections in the auditory system are frequency specific and localized ${ }^{13,16,17}$ connectivity profiles

108 between consecutive layers are modeled by a Gaussian profile of unknown connectivity width 109 parameter ${ }^{18}$ (Fig. 1e; specified by the $\mathrm{SD}, \sigma_{l}$ ). Finally, the sensitivity and firing rates of each layer 110 are controlled by adjusting the spike threshold level $\left(N_{l}\right)$ of each IF neuron ${ }^{19}$. This parameter 111 controls the firing pattern from a high firing rate dense code as proposed for the auditory periphery 
112 to a sparse code as has been proposed for auditory cortex ${ }^{2,20}$. Because temporal and spectral

113 selectivities vary systematically and gradually across auditory nuclei ${ }^{3,6,21}$, we required that the

114 network parameters vary hierarchically and smoothly from layer-to-layer according to (see

115 Methods: Network Constraints and Optimization)

116

117

118

119

120

121

122

123

124

125

126

127

128

129

130

131

132

133

134

135

136

$$
\begin{aligned}
& \tau_{l}=\tau_{1} \cdot \alpha^{l-1} \\
& \sigma_{l}=\sigma_{1} \cdot \gamma^{l-1} \\
& N_{l}=N_{1} \cdot \lambda^{l-1}
\end{aligned}
$$

where $\tau_{1}, \sigma_{1}$, and $N_{1}$ are the parameters of the first network layer and are chosen so that first layer responses mimic activity in auditory nerve fibers (see Methods). The scaling parameters $\alpha, \lambda$, and $\gamma$ determine the direction and magnitude of layer-to-layer changes for each of the three neuron parameters. Scaling values greater than one indicate that the neuron parameter increases systematically across layers, a value of one indicates that the parameter is constant, while a value less than one indicates that the parameter value decreases systematically across layers.

The optimal network outputs preserve important time-frequency information in speech despite variability in the input sound. Sounds in the optimization and validation corpus consist of spoken words for digits from zero to nine from eight talkers (TI46 LDC Corpus ${ }^{22}$, see Methods). As a task we require that the network identify the word (i.e., the digit) that is delivered as input (10 alternative forced choice task). Example cochlear model spectrograms and the network spiking outputs are shown in Fig. 1g and $\mathbf{h}$ for the words zero, six, and eight in the presence of speech babble noise (optimal outputs at $\mathrm{SNR}=20 \mathrm{~dB}$ ). Analogous to auditory cortex responses for speech ${ }^{7}$, the network produces a distinguishable spiking output for each sound that reflects its spectrotemporal composition (Fig. 1g). Furthermore, when a single word is generated by different talkers in noise $(\mathrm{SNR}=20 \mathrm{~dB})$ the network produces a relatively consistent firing pattern (Fig. 1g) such that the response timing and active neuron channels remain relatively consistent. For instance, a 
137 lack of activity is observed for neurons between $\sim 2-4 \mathrm{kHz}$ within the first $\sim 100-200 \mathrm{~ms}$ of the

138 sound for the word zero and several time-varying response peaks indicative of the vowel formants

139 are observed for all three talkers (Fig. 1h).

140 To determine the network architecture required for optimal word recognition in noise and

141 to identify whether such a configuration is essential for noise robust performance, we searched for

142 the network scaling parameters $(\alpha, \lambda$, and $\gamma)$ that maximize the network's word recognition

143 accuracy in a ten-alternative forced choice task for multiple talkers (8) and in the presence of

144 speech babble noise (signal-to-noise ratios, $\mathrm{SNR}=-5,0,5,10,15,20 \mathrm{~dB}$; see Methods). For each

145 input sound, the network spike train outputs are treated as response feature vectors and a Bayesian

146 classifier (Fig. 1c; see Methods) is used to read the network outputs and report the identified digit

147 (zero to nine). The network word recognition accuracy is shown in Fig. 2 as a function of each of

148 the network parameters $(\alpha, \lambda$, and $\gamma)$ and $\mathrm{SNR}(\mathbf{a}, \mathrm{SNR}=5 \mathrm{~dB} ; \mathbf{b}, \mathrm{SNR}=20 \mathrm{~dB}$; $\mathbf{c}$, average accuracy

149 across all SNRs). At each SNR the word recognition accuracy profiles are tuned with the scaling

150 parameter (i.e., concave function) which enables us to find an optimal scaling parameters that

151 maximizes the classifier performance. Regardless of the SNR the optimal HSNN parameters are

152 relatively constant (Fig. 2d; tested between -5 to $20 \mathrm{~dB}$ ) implying that the network organization is

153 relatively stable and invariant of the SNR (Fig. 2a-c; $\mathbf{a}=5 \mathrm{~dB}$ SNR, $\mathbf{b}=20 \mathrm{~dB}$ SNR, $\mathbf{c}=$ average

154 across all SNRs). Intriguingly, several functional characteristics of the optimal network mirror

155 those observed in the auditory pathway. Like the ascending auditory pathway where synaptic

156 potential time-constants vary from sub-millisecond in the auditory nerve to tens of milliseconds in

157 cortex $^{13,23-25}$, time constants scale in the optimal HSNN (global optimal $\alpha=1.9$ ) over more than

158 an order of magnitude between the first and last layer $\left(1.9^{5}=24.8\right.$ fold increase between the first

159 and last layer; $\sim 0.5$ to $12.5 \mathrm{~ms}$ ) indicating that temporal resolution becomes progressively coarser 
in the deep network layers. By comparison, the optimal connectivity widths do not change across

161 layers $(\gamma=1.0)$. This result suggests that for the optimal HSNN temporal resolution changes

162 dramatically while spectral resolution remains relatively constant across network layers, mirroring

163 changes in spectral and temporal selectivity observed along the ascending auditory pathway ${ }^{3-6}$. 1.9) and no change in connectivity resolution $(\gamma=1.0)$ across network layers. This prompted us

to ask how feature selectivity changes across the network layers and whether a sequential transformation in spectral and temporal selectivity is essential for optimal word recognition in noise. To quantify the sequential transformations in acoustic processing, we first measure the spectro-temporal receptive fields (STRFs) of each neuron in the network (see Methods). Example STRFs are shown for two selected frequencies across the six network layers (Fig. 3a; best

171 frequency $=1.5$ and $3 \mathrm{kHz}$ ). As a comparison, example STRFs from the auditory nerve (AN) ${ }^{26}$, 172 midbrain (inferior colliculus, IC) ${ }^{5}$, thalamus (MGB) and primary auditory cortex (A1) ${ }^{6}$ of cats

173 are shown in Fig. 3e. Like auditory pathway neurons, STRFs from the optimal HSNN contain 174 excitatory domains (red) with temporally lagged and surround inhibition/suppression (blue) along 175 the frequency dimension (Fig. 3a). Furthermore, STRFs are substantially faster in early network 176 layers lasting only a few milliseconds and mirroring STRFs from the auditory nerve, which have 177 relatively short latencies and integration times. STRFs have progressively longer integration times 178 (paired t-test with Bonferroni correction, $\mathrm{p}<0.01$; Fig. 3b) and latencies (paired t-test with 179 Bonferroni correction, $\mathrm{p}<0.01$; Fig. 3c) across network layers, while bandwidths increase only 180 slightly from the first to last layer (paired t-test with Bonferroni correction, $p<0.01$; Fig. 3d). These 181 sequential transformations mirror changes in temporal and spectral selectivity seen between the 182 auditory nerve, midbrain, thalamus and ultimately auditory cortex (Fig. 3e-h). As for the auditory 
183

184

185

186

187

188

189

190

191

192

193

194

195

196

197

198

199

200

201

202

203

204

205

network model, integration times (Fig. 3f) and latencies (Fig. 3g) increase systematically and smoothly (paired t-test with Bonferroni correction, $\mathrm{p}<0.01$ ) while bandwidths show a small but significant increase between the auditory nerve and cortex (paired t-test with Bonferroni correction, $\mathrm{p}<0.01$ ), analogous to results from the computational network. Although the network trends mirror changes in spectral and temporal selectivity seen between auditory nerve and cortex, auditory receptive fields tend to be somewhat slower and narrower than the network. Such disparities may partly be attributed to mechanisms not included in the HSNN such as descending feedback ${ }^{27}$, synaptic and dendritic nonlinearities ${ }^{28}$ and adaptive mechanisms such as spike time dependent plasticity, synaptic depression, and gain normalization ${ }^{1,29}$.

\section{Hierarchical and nonlinear transformations enhance robustness}

It is intriguing that the hierarchical loss of temporal and spectral resolution in the optimal network mirror changes in selectivity observed in the ascending auditory system, as this ought to limit the transfer of acoustic information across the network. One plausible hypothesis is that such a sequential decrease in resolution is necessary to extract invariant acoustic features in speech while rejecting noise and fine details in the acoustic signal that may contribute in a variety of hearing tasks (e.g., spatial hearing, pitch perception etc.), but ultimately don't contribute to speech recognition performance. This may be expected since human listeners require a limited set of temporal and spectral cues for speech recognition ${ }^{14,15}$ and can achieve high recognition performance even when spectral and temporal resolution is degraded ${ }^{30,31}$. We thus tested the above hypothesis by comparing the optimal network performance against a high-resolution network that lacks scaling ( $\alpha=1, \lambda=1$, and $\gamma=1)$ and for which we expect a minimal loss of acoustic information across layers. Unlike the optimal network, STRFs from the high-resolution network 
206

207

208

209

210

211

212

213

214

215

216

217

218

219

220

221

222

223

224

225

226

227 228

are relative consistent and change minimally across layers (Supplemental Data, Fig. 1S), which supports the idea that spectrotemporal information propagates across the high-resolution network with minimal processing.

Figure 4 illustrates how the optimal HSNN accentuates critical spectral and temporal cues necessary for speech recognition while the high-resolution network fails to do the same. Example Bayesian likelihood time-frequency histograms (average firing probability across all excerpts of each sound at each time-frequency bin) measured at 5 dB SNR are shown for the words "three", "four", "five" and "nine" for both the high-resolution (Fig. 4a) and optimal (Fig. 4b) HSNN along with selected spiking outputs from a single talker. Intriguingly, the Bayesian likelihood for the high-resolution network are highly blurred in both the temporal and spectral dimensions and have similar structure for the example words (Fig. 4a, right panels). This is also seen in the individual network outputs where the high-resolution network produces a dense and saturated firing pattern (Fig. 4a) that lacks the detailed spatio-temporal pattern seen in the optimal HSNN (Fig. 4b). The optimal HSNN preserves and even accentuates key acoustic elements such as temporal transitions for voice onset timing and spectral resonances (formants) while simultaneously rejecting and filtering out the background noise (Fig. 4b, right panels).

We next compared the performance of the HSNN models to human subjects in an isolated monosyllabic word recognition task in speech babble noise ${ }^{32}$. The word recognition accuracy of the optimal HSNN approaches human performance and is significantly higher than the highresolution network for all of the SNRs tested (Fig. 4 c; green=human subjects ${ }^{32}$; $p<0.001$, t-test with Bonferroni correction). On average there is a $27.6 \%$ improvement in the word accuracy rates for the optimal HSNN over the high-resolution HSNN. We also compared the accuracy of the optimal HSNN with the accuracy of a HSNN that was optimized individually at each SNR (SNR- 
optimal HSNN). The accuracy of the SNR-optimal HSNN was not significantly different from the optimal HSNN ( $<<0.05$, t-test $)$ which suggest that the optimal solution produces a stable noise

231 robust representation. Furthermore, the optimal HSNN is on average within 11.5\% of human

232 performance in an isolated word recognition task and follows a similar performance trend across 233 signal-to-noise ratios (Fig. 4c) ${ }^{32}$.

234 To characterize the neural transformations enabling noise robust coding, we examine how 235 acoustic information propagates and is transformed across sequential network layers. For each 236 layer, the spike train outputs are first fed to the Bayesian classifier in order to measure sequential 237 changes in word recognition accuracy. In the optimal HSNN, word recognition accuracy 238 systematically increases across layers with an average improvement of $15.5 \%$ between the first 239 and last layer when tested at $5 \mathrm{~dB}$ SNR ( $<<0.001$, t-test; Fig. 5a, blue; 13.7\% average improvement 240 across all SNRs). By comparison, for the high-resolution HSNN, performance degrades 241 sequentially across layers with an average decrease of $19.8 \%$ between the first and last layer 242 ( $<<0.001$, t-test; Fig. 5a, red; $18.1 \%$ average reduction across all SNRs). Thus, the optimal HSNN 243 is capable of sequentially extracting high-level acoustic features that enhance word recognition 244 performance in the presence of noise. In contrast, background noise persists in the spiking activity 245 of the high-resolution network, which results in a greater performance reduction across network 246 layers.

248 optimal HSNN, a similar trend is not observed for the transfer of acoustic information. First, firing 249 rates decrease systematically across layers for the optimal HSNN, consistent with a sparser output 250 representation (Fig. 5b, blue) as proposed for deep layers of the auditory pathway ${ }^{2,20,33}$. By 251 comparison, firing rates are relatively stable across layers for the high-resolution network (Fig. 5b, 
252 red). We next measure the average mutual information (see Methods) in the presence of noise (5

$253 \mathrm{~dB}$ ) to identify how incoming acoustic information is sequentially transformed from layer-to-layer.

254 For the optimal HSNN the information rates (i.e., bits / sec) decreases between the first and last

255 layer (Fig. 5c, blue) whereas for the high-resolution network information is conserved across

256 network layers (Fig. 5c, red). Thus, the layer-to-layer increase in word recognition accuracy

257 observed for the optimal HSNN is accompanied by a loss of total acoustic information in the deep

258 network layers. We next measure the average information conveyed by individual action potentials

259 as way of determining how acoustic features are represented by individual precisely timed spikes.

260 Surprisingly, the information conveyed by single action potentials is higher and increases across

261 layers (Fig. 5d, blue). This contrast the high-resolution HSNN where information per spike

262 remains relatively constant across layers (Fig. 5d, red). This indicates that individual action

263 potentials become increasingly more informative from layer-to-layer in the optimal HSNN despite

264 a reduction in firing rates. Taken together with the changes in spectro-temporal selectivity (Fig.

2653 ), the findings are consistent with the hypothesis that the optimal HSNN produces a noise resilient

266 sparse code in which invariant acoustic features are represented with isolated spikes. By

267 comparison, the high-resolution network produces a dense response pattern that has a tendency to

268 preserve incoming acoustic information, including the background noise and nonessential acoustic

269 features, thus suffering in recognition performance.

271 are required for robust coding of speech. Hypothetically, its plausible that similar performance

272 could be achieved with a single layer network as long as each neuron accounts for the overall

273 network receptive field transformations. To test this, we developed single-layer networks

274 consisting of generalized linear model neurons ${ }^{34}$ with either a linear receptive field and Poisson 
275 spike train generator (LP network) or a linear receptive field and nonlinear stage followed by

276 Poisson spike train generator (LNP network) (Fig. 6a; see Methods). The performance of the LP

277 network, which accounts for the linear transformations of the optimal HSNN, was on average

$27821.7 \%$ lower than the optimal HSNN indicating that nonlinearities are critical to achieve high word

279 recognition accuracy (Fig. 6b). Its plausible that this performance disparity can be overcome by

280 incorporating a nonlinearity that models the rectifying effects in the spike generation process of

281 neurons (LNP network). Doing so improves the performance to within $2.1 \%$ of the optimal HSNN

282 when there is little background noise ( $\mathrm{SNR}=20 \mathrm{~dB}, 85.6 \%$ for optimal HSNN versus $82.5 \%$ for

283 LNP network). However, the performance degraded when background noise was added when

284 compared to the optimal HSNN, with an overall performance reduction of $13.8 \%$ at $-5 \mathrm{~dB}$ SNR

285 (58.4\% for optimal HSNN versus $44.6 \%$ for LNP network).

The robustness of each network was next examined by comparing the performance of each

287 model against human performance trends. For each condition, we measured the relative accuracy

288 change (RAC) between the model and human performance (Methods, Fig. 6c). The RAC of the

289 optimal HSNN was near zero with a small reduction in RAC of only $3.9 \%$ at $-5 \mathrm{~dB}$ SNR. Thus,

290 the optimal HSNN follows a similar trend as humans across background noise levels. By

291 comparison, both the LP and LNP performance diverged from human performance with increasing

292 background noise with an overall RAC reduction of $22.2 \%$ and $15.6 \%$ at $-5 \mathrm{~dB}$ SNR, respectively.

293 Thus, in contrast to the optimal HSNN trends which mirrors human data, the LP and LNP network

294 performance diverged from the human trend with increasing background noise.

The average performance of each network was also compared against human word

296 recognition accuracy. The accuracy for the optimal and SNR optimal HSNNs are not significantly

297 differences when compared against human accuracy rates with an average reduction of 9.7\% and 
$11.5 \%$, respectively ( $>0.05$, t-test). Furthermore, the optimal HSNN outperformed all other models tested. The LNP, LP, and high-resolution HSNN exhibited a rank order reduction in performance relative to human accuracy $(18.5 \%, 33.3 \%, 37.2 \%$ respectively; $\mathrm{p}<0.05$, t-test with

301

302

303

304

305

306

307

308

309

310

311

312

313

314

315

316

317

318

319

320

Bonferroni Correction).

Overall, the findings indicate that although the linear and nonlinear receptive field transformations both contribute to the overall network performance, the sequential layer-to-layer transformations carried out by the optimal HSNN are critical for maintaining a noise robust representation that mirrors human performance trends.

\section{Optimal spiking timing resolution}

Finally, we identified the spike timing resolution required to maximize recognition accuracy as previously identified when "reading out" neural activity in auditory cortex ${ }^{7,35}$. To do so, we synthetically manipulating the temporal resolution of the output spike trains while measuring the word recognition accuracy at multiple SNRs (see Methods). An optimal spike timing resolution is identified within the vicinity of 4-14 ms for the optimal network (Fig. 7a and b) which is comparable to spike timing precision required for sound recognition in auditory cortex 7,35. By comparison, the high-resolution network requires a high temporal resolution of $\sim 2 \mathrm{~ms}$ to achieve maximum word accuracy (46.6\% accuracy across all SNRs; Fig. 8c), which is $\sim 31.8 \%$ lower on average than the optimal network $(78.4 \%$ accuracy for the optimal HSNN across all SNRs). Taken across all SNRs, the optimal temporal resolution that maximized word accuracy rates is $6.5 \mathrm{~ms}$, which is comparable to the spike timing resolution reported for optimal speech and vocalizations recognition in auditory cortex ${ }^{7,35}$. 


\section{Discussion}

323 is consistent with a near optimal strategy for feature extraction that maximizes sound recognition

324 performance and is relatively impervious to noise. Upon optimizing the network organization on

325 a behaviorally relevant word recognition task, the HSNN achieves high recognition accuracy and

326 follows a similar noise robust trend that is within $\sim 10 \%$ of human performance by sequentially

327 refining the spectral and temporal selectivity from layer-to-layer. Similar noise robustness is not

328 replicated with conventional receptive field based networks even when the receptive fields capture

329 the linear integration of the optimal HSNN and a threshold nonlinearity was imposed. The

330 sequential nonlinear transformations of the optimal HSNN preserve critical acoustic features for

331

332

333

334

335

336 speech recognition while simultaneously discarding acoustic noise not relevant to the sound recognition task. These transformations mirror changes in selectivity along the ascending auditory pathway, including an extensive loss of temporal resolution ${ }^{3}$, slight loss of spectral resolution ${ }^{4-6}$, and increase in sparsity ${ }^{2,20}$. The simulations suggest that the orderly arrangement of receptive fields and sequential nonlinear transformations of the ascending auditory pathway may be critical to achieve a noise robust code.

Critical to our findings is the observation that the optimal network transformations described here are not expected a priori as a general sensory processing strategy and may in fact be unique to audition. For instance, changes in temporal selectivity between the retina, visual thalamus, and visual cortex are generally small and neurons in the visual pathway synchronize over a relatively narrow range of frequencies (typically $<20 \mathrm{~Hz}$ ) ${ }^{36-39}$. This differs dramatically from the observed increase in integration times reported here, systematic increase in synaptic potential time-constants ${ }^{13,23-25}$, and a corresponding reduction in synchronization ability ${ }^{3}$ 
344 observed between the auditory nerve and auditory cortex. By comparison, in the spatial domain,

345 there is substantial divergence in connectivity between the retina and visual cortex since visual

346 receptive fields sequentially grow in size between the periphery and cortex so as to occupy a larger

347 area of retinotopic space ${ }^{40-42}$. This contrasts changes in frequency receptive fields in which only

348 a subtle increase in average bandwidth is observed between the auditory nerve and cortex ${ }^{4-6,21,26}$,

349 consistent with findings from the optimal sound recognition strategy.

351 achieving high recognition accuracy, particularly in the presence of noise. Although the auditory

352 pathway is substantially more complex than the proposed HSSN, which lacks anatomical elements

353 such as the binaural circuits in the brainstem and descending feedback, it is nonetheless surprising

354 that the optimal strategy for speech recognition replicates sequential transformations observed

355 along the auditory pathway. Furthermore, whereas auditory receptive fields can be more diverse

356 than those of the HSNN, the receptive fields of the optimal HSSN nonetheless contain basic

357 features seen across the auditory pathway including lateral inhibition, temporal inhibition or

358 suppression, and sequentially increasing time-constants along the hierarchy ${ }^{6,26,43-45}$. The HSSN

359 employs several computational principles observed anatomically and physiologically, including

360 the presence of spiking neurons, inhibitory connections, cotuning between excitation and

361 inhibition, and a frequency specific localized circuitry, all of which likely contribute to its high

362 performance. Furthermore, these sequential transformations appear to be critical since single layer

363 generalized linear models designed to capture the overall transformations of the HSNN did not

364 achieve comparable levels of performance.

Recent advances in deep neural networks (DNN) have made it possible to achieve high-

366 levels of speech recognition performance approaching human performance limits ${ }^{46,47}$. Yet, these 
networks typically require tens-of-thousands of neurons and parameters to do so and the mechanisms leading to high recognition accuracy are based on neuron elements designed on principles of rate coding. The HSNN developed here, by comparison, employs temporal coding and organizational principles identified physiologically and approaches human performance levels

371 with just 600 neurons and three meta-parameters that control the layer-to-layer transformations.

372 Like the auditory pathway, the auditory HSNN is inherently temporal as it contains spiking 373 neurons capable of precisely synchronizing to the sound features and exhibit hierarchical changes

374 in time-scale across layers observed physiologically ${ }^{3}$. Furthermore, whereas DNNs rely on strictly excitatory connection weights between neuron, feature extraction in the HSNN is shaped by both excitatory and inhibitory circuitry as observed in central auditory structures ${ }^{10-13}$. A challenge for

377 future studies is to further reveal biologically realistic strategies for auditory signal processing,

378 feature extraction, and classification, including descending feedback ${ }^{27}$ and adaptive mechanisms

$379^{1,29}$, that together endow perceptual capabilities for sound recognition and promote robust coding.

\section{$381 \quad$ Materials and Methods}

382 Speech Corpus: Sounds in the experimental dataset consist of isolated digits (zero to nine) from 383 eight male talkers from LDC TI46 corpus $^{22}$. Ten utterances for each digit are used for a total of 384800 sounds ( 8 talkers $\times 10$ digits/subject $x 10$ utterances/digit). Words are temporally aligned based 385 on the waveform onset (first upward crossing that exceeds 2 SD of the background noise level) 386 and speech babble noise (generated by adding 7 randomly selected speech segments) is added at 387 multiple signal-to-noise ratios $(\mathrm{SNR}=-5,0,5,10,15$ and $20 \mathrm{~dB})$. This range of SNR was selected 388 to allow comparisons with human isolated word recognition performance in the presence of speech 389 babble noise ${ }^{32}$. 
391 Auditory Model and Hierarchical spiking neural Network (HSNN): We developed a multi-

392 layer auditory network model consisting of a cochlear model stage containing gamma tone filters $393\left(0.1-4 \mathrm{kHz}\right.$; center frequencies $1 / 10^{\text {th }}$ octave separation; critical band resolution), envelope 394 extraction and nonlinear compression ${ }^{48}$ followed by a HSNN as illustrated in Fig. 1. Several 395 architectural and functional constraints are imposed on the spiking neural network to mirror 396 auditory circuitry and physiology. First, the network contains six layers as there are six principal 397 nuclei between the cochlea and cortex. Second, connections between consecutive layers contain 398 both excitatory and inhibitory projections since long-range inhibitory projections between nuclei 399 are pervasive in the ascending auditory system ${ }^{10,49}$. Each layer in the network contains 53 400 excitatory and 53 inhibitory frequency organized neurons per layer which allows for $1 / 10^{\text {th }}$ octave 401 resolution over the frequency range of the cochlear model $(0.1-4 \mathrm{kHz})$. Furthermore, since 402 ascending projections in the central auditory pathway are spatially localized and frequency specific $40318,49,50$, excitatory and inhibitory connection weights are modeled by co-tuned Gaussian profiles of 404 unspecified connectivity width (Fig. 1e):

405

$$
w_{l, m, n}^{E}=\frac{1}{\sqrt{2 \pi \sigma_{E}^{2}}} \cdot e^{-\left(x_{l, m}-x_{l+1, n}\right)^{2} / 2 \sigma_{E}^{2}}
$$

407

$$
w_{l, m, n}^{I}=\frac{1}{\sqrt{2 \pi \sigma_{I}^{2}}} \cdot e^{-\left(x_{l, m}-x_{l+1, n}\right)^{2} / 2 \sigma_{I}^{2}}
$$

409 where $w_{l, m, n}^{I}$ and $w_{l, m, n}^{E}$ are the inhibitory and excitatory connection weights between the m-th 410 and n-th neuron from layer $l$ and $l+1, x_{l, m}$ and $x_{l+1, n}$ are the normalized spatial positions $(0-1)$

411 along the frequency axis of the $m$-th and $n$-th neurons in layers $l$ and $l+1$, and $\sigma_{I}$ and $\sigma_{E}$ are the 
412 inhibitory and excitatory connectivity widths (i.e., SD of Gaussian connection profiles), which

413 determine the spatial spread and ultimately the frequency resolution of the ascending connections.

414 Each neuron in the network consists of a modified leaky integrate-and-fire (LIF) neuron ${ }^{51}$

415 receiving excitatory and inhibitory presynaptic inputs (Fig. 1e). Given a presynaptic spike trains

416 from the $m$-th neurons in network layer- $l\left(s_{l, m}(t)\right)$ the desired intracellular voltage of the $n$-th

417 neuron in network layer $l+1$ is obtained as

418

$$
v_{l+1, n}(t)=\sum_{m} w_{l, m, n}^{E} \cdot h_{E P S P}(t) * s_{l, m}(t)-\beta \sum_{m} w_{l, m, n}^{I} \cdot h_{I P S P}(t) * s_{l, m}(t)
$$

422 inhibitory currents, $h_{E P S P}(t)$ and $h_{I P S P}(t)$ are temporal kernels that model excitatory and

423 inhibitory post synaptic potentials generated for each incoming spike as an alpha function (Fig. 1e,

424 red and blue curves) $)^{51}$. Since central auditory receptive fields often have extensive lateral

425 inhibition/suppression beyond the central excitatory tuning area and inhibition is longer lasting 426 and weaker ${ }^{5,6}$ we require that $\sigma_{I}=1.5 \cdot \sigma_{E}, \tau_{I}=1.5 \cdot \tau_{E}$, and $\beta=2 / 3$, as this produced realistic

427 receptive field measurements. For simplicity, we use $\sigma$ and $\tau$ interchangeably with $\sigma_{E}$ and $\tau_{E}$,

428 since these determine the overall spectral and temporal resolution of each neuron.

429 Because the input to an LIF neuron is a current injection, we derived the injected current by deconvolving the LIF neuron time-constant from the desired membrane voltage 
434 where $i_{l+1, n}(t)$ is the injected current for the $n$-th neuron in layer $l+1$ and $v_{l+1, n}(t)$ is the 435 corresponding output voltage and $z(t)$ is a noise current component. As we demonstrated 436 previously ${ }^{19}$, this procedure removes the influence of the cell membrane integration prior to

437 injecting the current in the IF neuron compartment and allows us to precisely control the

438 intracellular voltage delivered to each LIF neuron. Above $h(t)=\frac{1}{C} e^{-t / \tau} u(t)$ is the impulse

439 response of the cell membrane $(u(t)$ is the step function), $\mathrm{C}$ is the membrane capacitance, $\tau$, is the

440 membrane time-constant and $h^{-1}(t)$ is the inverse kernel (i.e., $h(t) * h^{-1}(t)=\delta(t)$ where $\delta(t)$

441 is the Diract function). Because the EPSP time constant and the resulting temporal resolution of

442 the intracellular voltage are largely influenced by the cell membrane integration, we require that

$443 \tau=\tau_{E}$. Finally, Gaussian white noise, $z(t)$, is added to the injected current in order to generate

444 spike timing variability (signal-to-noise ratio $=15 \mathrm{~dB}$ ) ${ }^{19}$. Upon injecting the current, the resulting 445 intracellular voltage follows $v_{l+1, n}(t)+z(t) * h(t)$ and the IF model generates spikes whenever

446 the intracellular voltage exceeds a normalized threshold value ${ }^{19}$. The normalized threshold is 447 specified for each network layer $(l)$ as

$$
N_{l}=\left(V_{T}-V_{r}\right) / \sigma_{V, l}
$$

451 where $V_{T}=-45 \mathrm{mV}$ is the threshold voltage, $V_{r}=-65 \mathrm{mV}$ is the membrane resting potentials,

452 and $\sigma_{V, l}$ is the standard deviation of the intracellular voltages for the population of neurons in layer

453 l. As demonstrated previously, this normalized threshold represents the number of standard

454 deviations the intracellular activity is away from the threshold activation and serves as a way of 455 controlling the output sensitivity of each network layer. Upon generating a spike, the voltage is 
reset to the resting potential, a $1 \mathrm{~ms}$ refractory period is imposed, and the membrane temporal

457 integration continues.

458

Decision model: The neural outputs of the network consist of a spatio-temporal spiking pattern bins. The number of time bins is dependent on the temporal resolution for each bin, $\Delta t$, which is varied between $0.5-100 \mathrm{~ms}$. Each response $\left(r_{n, i} ; n-\right.$ th neuron and $i-$ th time bin $)$ is assigned a 1 or 0 value indicating the presence or absence of spikes, respectively. and categorize individual speech words. The classified digit $(y)$ is the one that maximizes posterior probability for a particular response according to

$$
y=\underset{d=\{0 \ldots 9\}}{\operatorname{argmax}} \prod_{n, i} p_{d, n, i}^{r_{n, i}} \cdot\left(1-p_{d, n, i}\right)^{1-r_{n, i}}
$$

Network Constraints and Optimization: The primary objective is to determine the spectral and temporal resolution of the network connections as well as the network sensitivity necessary for

477 robust speech recognition. Specifically, we hypothesize that the temporal and spectral resolution 478 and sensitivity of each network layer need to be hierarchically organized across network layers in 
479 order to maximize speech recognition performance in the presence of noise. We thus optimize

480 three key parameters, the time constant $\left(\tau_{l}\right)$, connectivity widths $\left(\sigma_{l}\right)$, and normalized threshold

$481\left(N_{l}\right)$ that separately control these functional attributes of the network, where the index $l$ designates

482 the network layer (1-6). Given that spectro-temporal selectivity changes systematically and

483 gradually between auditory nuclei, we constrained the parameters to vary smoothly from layer-to-

484 layer according to the power law rules of Eqn. 1. The initial parameters for the first network layer,

$485 \tau_{1}=0.4 \mathrm{~ms}, \sigma_{1}=0.0269$ (equivalent to $\sim 1 / 6$ octave), and $N_{1}=0.5$, are selected to allow for

486 high-temporal and spectral resolution and high firing rates, analogous to physiological

487 characteristics of auditory nerve fibers ${ }^{3,4,26}$ and inner hair cell ribbon synapse ${ }^{23}$. We optimize for

488 the three scaling parameters $\alpha, \lambda$, and $\gamma$, which determine the direction and magnitude of layer-to-

489 layer changes and ultimately the network organization rules for temporal and spectral resolution

490 and network sensitivity.

The optimization is carried using a cross-validation grid search procedure in which we maximized word accuracy rates (WAR). Initial tests are performed to determine a suitable search

493 range for the scaling parameters and a final global search is performed over the resulting search space $(\alpha=0.9-2.3, \lambda=0.5-1.6$ and $\gamma=0.8-1.5 ; 0.1$ step size for all parameters $)$. For each

495 parameter combination, the network is required to identify the digits in the speech corpus with a 496 ten-alternative forced choice task. For each iteration we select one utterance from the speech 497 corpus ( 1 of 800 ) for validation and use the remaining utterances (799) to train the model by 498 deriving the Bayesian likelihood functions (i.e., $p_{d, n, i}$ ). The Bayesian classifier is then used to 499 identify the validation utterances and compute WAR for that iteration (either 0 or $100 \%$ for each 500 iteration). This procedure is iteratively repeated 800 times over all of the available utterances and 501 the overall WAR is computed as the average over all iterations. This procedure is also repeated for 
502 five distinct signal-to-noise ratios $(\mathrm{SNR}=-5,0,5,10,20 \mathrm{~dB})$. Example curves showing the WAR

503 as a function of scaling parameters and SNR are shown in Fig. 2 (a and b, shown for 5 and 20dB).

504 The global optimal solution for the scaling parameters is obtained by averaging WAR across all

505 SNRs and selecting the scaling parameter combinations that maximize the WAR (Fig. 2c).

506

507 Receptive Field and Mutual Information Calculation: To characterize the layer-to-layer

508 transformations performed by the network, we compute spectro-temporal receptive fields (STRFs)

509 and measure the mutual information conveyed by each neuron in the network. First, STRFs are

510 obtained by delivering dynamic moving ripple sounds (DMR), which are statistically unbiased,

511 and cross-correlating the output spike trains of each neuron with the DMR spectrotemporal

512 envelope ${ }^{53}$. For each STRF, we estimate the temporal and spectral resolution by computing the

513 integration time and bandwidths, as described previously ${ }^{5}$. Mutual information is calculated by

514 delivering a sequence of digits ( 0 to 9 ) at $5 \mathrm{~dB}$ SNR to the network. The procedure is repeated 50

515 trials with different noise seeds and the spike trains from each neuron are converted into a dot-

516 raster sampled at $2 \mathrm{~ms}$ temporal resolution. The mutual information is calculated for each neuron

517 in the network using the procedure of Strong et al. ${ }^{54}$ as described previously ${ }^{19}$.

519 Auditory System Data: Previously published data from single neurons in the auditory nerve $520(n=214)^{26}$, auditory midbrain (Central Nucleus of the Inferior Colliculus, $\left.n=125\right)^{48}$, thalamus

521 (Medial Geniculate Body, $n=88$ ) and primary auditory cortex $(n=83)^{6}$ is used to quantify

522 transformations in spectral and temporal selectivity between successive auditory nuclei. Using the

523 measured spectro-temporal receptive fields of each neuron (Fig. 3), the spectral and temporal

524 selectivity are quantified by computing integration times, response latencies, and bandwidths as 
525 described previously ${ }^{5}$. Sequential changes in selectivity across ascending auditory nuclei are 526 summarized by comparing the neural integration parameters of each auditory structure (Fig. 3f-h).

Generalized Linear Model (GLM) Networks: To identify the role of linear and nonlinear receptive field transformations for noise robust coding, we developed two single-layers networks containing GLM neurons $^{34}$ (Fig. 6a) that are designed to capture linear and nonlinear transformations of the HSNN.

534 For each output of the optimal network ( $m$-th output) we measured the STRF and fitted it to a

535 Gabor model $\left(\operatorname{STRF}_{m}\left(t, f_{k}\right)\right)^{43}$. On average the fitted Gabor model accurately replicated the 536 structure in the measured STRFs and on average accounted for 99\% of the STRF variance (range 537 94-99.9\%). The output firing rate of the $m$-th LP model neuron is obtained as

$$
\lambda_{m}(t)=\lambda_{0}+G \cdot \sum_{k=1}^{N} S\left(t, f_{k}\right) * \operatorname{STRF}_{m}\left(t, f_{k}\right)
$$

541 where $S\left(t, f_{k}\right)$ is the cochlear model output, * is the convolution operator, $G$ is a gain term, and $\lambda_{0}$ 542 is required to assure that the spike rates are strictly positive and the firing maintains a linear 543 relationship with the sound. $G$ and $\lambda_{0}$ are chosen so that the average firing rate taken across all 544 output neurons and sounds matches the average firing rate of the optimal network and are strictly 545 greater than zero. The firing rate functions for each channel, $\lambda_{m}(t)$, are then passed through a 546 nonhomogenous Poisson point process in order to generate the spike trains for each output channel. 
Next we explored the role of nonlinear rectification by incorporating a rectification stage in the LP model. The firing of the m-th neuron in the LNP (linear nonlinear Poisson) network is

$$
\lambda_{m}(t)=G \cdot \max \left[0, \sum_{k=1}^{N} S\left(t, f_{k}\right) * \operatorname{STRF}_{m}\left(t, f_{k}\right)\right]
$$
and all words matches the average firing rate of the optimal HSNN. accuracy rate values were estimated for human subjects at the model conditions tested. The

564 relative accuracy change $\mathrm{RAC}=\left(\mathrm{A}_{\text {model }}-\mathrm{A}_{\text {human }}\right)-\left(\mathrm{A}_{\text {model }}^{20 \mathrm{~dB}} \mathrm{~A}^{20 \mathrm{~dB}}\right.$ human $)$ was used to measure the

565 divergence of each model across SNR when compared against human accuracy rates (i.e., Fig. 6c).

566 An RAC of 0 indicates that the model performance follows a similar noise robust trend when

567 compared to humans. Values $<0$ indicate that the model accuracy deviated (in units of $\%$ ) from the 568 human trend. 


\section{REFERENCES}

5721 Mesgarani, N., David, S. V., Fritz, J. B. \& Shamma, S. A. Mechanisms of noise robust 573 representation of speech in primary auditory cortex. Proc Natl Acad Sci U S A 111, 6792-6797, doi:10.1073/pnas.1318017111 (2014).

576

Schneider, D. M. \& Woolley, S. M. Sparse and background-invariant coding of vocalizations in auditory scenes. Neuron 79, 141-152,

579 doi:10.1016/j.neuron.2013.04.038 (2013).

3 Joris, P. X., Schreiner, C. E. \& Rees, A. Neural processing of amplitude-modulated

581 sounds. Physiol Rev 84, 541-577 (2004).

4 Mc Laughlin, M., Van de Sande, B., van der Heijden, M. \& Joris, P. X. Comparison of bandwidths in the inferior colliculus and the auditory nerve. I. Measurement using a spectrally manipulated stimulus. J Neurophysiol 98, 2566-2579 (2007).

5835 Rodriguez, F. A., Read, H. L. \& Escabi, M. A. Spectral and temporal modulation tradeoff in the inferior colliculus. J Neurophysiol 103, 887-903, doi:10.1152/jn.00813.2009 (2010).

587

588

6 Miller, L. M., Escabi, M. A., Read, H. L. \& Schreiner, C. E. Spectrotemporal receptive fields in the lemniscal auditory thalamus and cortex. J Neurophysiol 87, 516-527 (2002).

7 Engineer, C. T. et al. Cortical activity patterns predict speech discrimination ability. Nat Neurosci 11, 603-608, doi:nn.2109 [pii]

\subsection{8/nn.2109 (2008).}

8 Sachs, M. B., Voigt, H. F. \& Young, E. D. Auditory nerve representation of vowels in background noise. J Neurophysiol 50, 27-45 (1983).

9 Delgutte, B. \& Kiang, N. Y. Speech coding in the auditory nerve: I. Vowel-like sounds. J Acoust Soc Am 75, 866-878 (1984).

10 Winer, J. A., Saint Marie, R. L., Larue, D. T. \& Oliver, D. L. GABAergic feedforward projections from the inferior colliculus to the medial geniculate body. Proc Natl Acad Sci U S A 93, 8005-8010 (1996).

600

601

602

603

604

605

606

607

11 Loftus, W. C., Bishop, D. C., Saint Marie, R. L. \& Oliver, D. L. Organization of binaural excitatory and inhibitory inputs to the inferior colliculus from the superior olive. $J$ Comp Neurol 472, 330-344 (2004).

12 Oswald, A. M., Schiff, M. L. \& Reyes, A. D. Synaptic mechanisms underlying auditory processing. Curr Opin Neurobiol 16, 371-376, doi:10.1016/j.conb.2006.06.015 (2006).

611

612

13 Wehr, M. \& Zador, A. M. Balanced inhibition underlies tuning and sharpens spike timing in auditory cortex. Nature 426, 442-446 (2003).

14 Elliott, T. M. \& Theunissen, F. E. The modulation transfer function for speech intelligibility. PLoS Comput Biol 5, e1000302, doi:10.1371/journal.pcbi.1000302 (2009).

15 Chi, T., Gao, Y., Guyton, M. C., Ru, P. \& Shamma, S. Spectro-temporal modulation transfer functions and speech intelligibility. J Acoust Soc Am 106, 2719-2732 (1999). 
641

642

643

644

645

646

647

648

649

650

651

652

653

654

655

656

657

658

01020.2003 [pii] (2004).

17 Xie, R., Gittelman, J. X. \& Pollak, G. D. Rethinking tuning: in vivo whole-cell recordings of the inferior colliculus in awake bats. J Neurosci 27, 9469-9481, doi:27/35/9469 [pii]

10.1523/JNEUROSCI.2865-07.2007 (2007).

18 Levy, R. B. \& Reyes, A. D. Spatial profile of excitatory and inhibitory synaptic connectivity in mouse primary auditory cortex. J Neurosci 32, 5609-5619, doi:10.1523/JNEUROSCI.5158-11.2012 (2012).

19 Escabi, M. A., Nassiri, R., Miller, L. M., Schreiner, C. E. \& Read, H. L. The contribution of spike threshold to acoustic feature selectivity, spike information content, and information throughput. J Neurosci 25, 9524-9534, doi:10.1523/JNEUROSCI.180405.2005 (2005).

20 Hromadka, T., Deweese, M. R. \& Zador, A. M. Sparse representation of sounds in the unanesthetized auditory cortex. PLoS biology 6, e16, doi:07-PLBI-RA-1814 [pii]

10.1371/journal.pbio.0060016 (2008).

21 Escabi, M. A. \& Read, H. L. Neural mechanisms for spectral analysis in the auditory midbrain, thalamus, and cortex. Int Rev Neurobiol 70, 207-252, doi:10.1016/S00747742(05)70007-6 (2005).

22 Liberman, M. e. a. (ed Linguistics Data Symposium) (NIST Speech Disc 7-1.1 (1 disc) 1991).

23 Grant, L., Yi, E. \& Glowatzki, E. Two modes of release shape the postsynaptic response at the inner hair cell ribbon synapse. J Neurosci 30, 4210-4220, doi:10.1523/JNEUROSCI.4439-09.2010 (2010).

24 Franken, T. P., Roberts, M. T., Wei, L., Golding, N. L. \& Joris, P. X. In vivo coincidence detection in mammalian sound localization generates phase delays. Nat Neurosci 18, 444-452, doi:10.1038/nn.3948 (2015).

25 Geis, H. R. \& Borst, J. G. Intracellular responses of neurons in the mouse inferior colliculus to sinusoidal amplitude-modulated tones. J Neurophysiol 101, 2002-2016, doi:90966.2008 [pii]

10.1152/jn.90966.2008 (2009).

26 Kim, P. J. \& Young, E. D. Comparative analysis of spectro-temporal receptive fields, reverse correlation functions, and frequency tuning curves of auditory-nerve fibers. J Acoust Soc Am 95, 410-422 (1994).

27 Suga, N. Role of corticofugal feedback in hearing. J Comp Physiol A Neuroethol Sens Neural Behav Physiol 194, 169-183, doi:10.1007/s00359-007-0274-2 (2008).

28 Reyes, A. Influence of dendritic conductances on the input-output properties of neurons. Annu Rev Neurosci 24, 653-675 (2001).

29 Rabinowitz, N. C., Willmore, B. D., Schnupp, J. W. \& King, A. J. Contrast gain control in auditory cortex. Neuron 70, 1178-1191, doi:S0896-6273(11)00435-1 [pii]

10.1016/j.neuron.2011.04.030 (2011).

30 Shannon, R. V., Zeng, F. G., Kamath, V., Wygonski, J. \& Ekelid, M. Speech recognition with primarily temporal cues. Science 270, 303-304 (1995).

31 Drullman, R., Festen, J. M. \& Plomp, R. Effect of temporal envelope smearing on speech reception. J Acoust Soc Am 95, 1053-1064 (1994). 
32 Crandell, C. C. \& Smaldino, J. J. Classroom Acoustics for Children With Normal Hearing and With Hearing Impairment. Lang Speech Hear Serv Sch 31, 362-370, doi:10.1044/0161-1461.3104.362 (2000).

33 Chen, C., Read, H. L. \& Escabi, M. A. Precise feature based time scales and frequency decorrelation lead to a sparse auditory code. J Neurosci 32, 8454-8468, doi:10.1523/JNEUROSCI.6506-11.2012 (2012).

34 Simoncelli, E. P., Paninski, L., Pillow, J. W. \& Schwartz, O. Characterization of Neural Responses with Stochastic Stimuli. The New Cognitive Neuroscience 3, 327-338 (2004).

35 Narayan, R., Grana, G. \& Sen, K. Distinct time scales in cortical discrimination of natural sounds in songbirds. J Neurophysiol 96, 252-258, doi:01257.2005 [pii] 10.1152/jn.01257.2005 (2006).

36 DeAngelis, G. C., Ohzawa, I. \& Freeman, R. D. Spatiotemporal organization of simplecell receptive fields in the cat's striate cortex. II. Linearity of temporal and spatial summation. J Neurophysiol 69, 1118-1135 (1993).

37 Cai, D., DeAngelis, G. C. \& Freeman, R. D. Spatiotemporal receptive field organization in the lateral geniculate nucleus of cats and kittens. J Neurophysiol 78, 1045-1061 (1997).

38 Derrington, A. M. \& Lennie, P. The influence of temporal frequency and adaptation level on receptive field organization of retinal ganglion cells in cat.J Physiol 333, 343-366 (1982).

39 Dawis, S., Shapley, R., Kaplan, E. \& Tranchina, D. The receptive field organization of $\mathrm{X}$-cells in the cat: spatiotemporal coupling and asymmetry. Vision Res 24, 549-564 (1984).

40 Motter, B. C. Central V4 receptive fields are scaled by the V1 cortical magnification and correspond to a constant-sized sampling of the V1 surface.J Neurosci 29, 57495757, doi:10.1523/JNEUROSCI.4496-08.2009 (2009).

41 Alonso, J. M., Usrey, W. M. \& Reid, R. C. Rules of connectivity between geniculate cells and simple cells in cat primary visual cortex. J Neurosci 21, 4002-4015 (2001).

42 Usrey, W. M., Reppas, J. B. \& Reid, R. C. Specificity and strength of retinogeniculate connections. J Neurophysiol 82, 3527-3540 (1999).

43 Qiu, A., Schreiner, C. E. \& Escabi, M. A. Gabor analysis of auditory midbrain receptive fields: spectro-temporal and binaural composition. J Neurophysiol 90, 456-476, doi:10.1152/jn.00851.2002

00851.2002 [pii] (2003).

44 Depireux, D. A., Simon, J. Z., Klein, D. J. \& Shamma, S. A. Spectro-temporal response field characterization with dynamic ripples in ferret primary auditory cortex. $J$ Neurophysiol 85, 1220-1234 (2001).

45 Sen, K., Theunissen, F. E. \& Doupe, A. J. Feature analysis of natural sounds in the songbird auditory forebrain. J Neurophysiol 86, 1445-1458 (2001).

46 Dahl, G. E., Yu, D., Deng, L. \& Acero, A. Context-dependent pre-trained deep neural networks for large-vocabulary speech recognition. IEEE Trans Audio, Speech and Language Processing 20, 30-42 (2011).

47 Hinton, G. et al. Deep neural networks for acoustic modeling in speech recognition: The shared views of four research groups. Signal Processing Magazine, IEEE 29, 8297 (2012). 
70548 Rodriguez, F. A., Chen, C., Read, H. L. \& Escabi, M. A. Neural modulation tuning

706 characteristics scale to efficiently encode natural sound statistics. J Neurosci 30,

707 15969-15980, doi:10.1523/JNEUROSCI.0966-10.2010 (2010).

70849 Oliver, D. L. Ascending efferent projections of the superior olivary complex. Microsc

$709 \quad$ Res Tech 51, 355-363 (2000).

$71050 \quad$ Read, H. L., Miller, L. M., Schreiner, C. E. \& Winer, J. A. Two thalamic pathways to

711 primary auditory cortex. Neuroscience 152, 151-159, doi:S0306-4522(07)01472-8

$712 \quad$ [pii]

713 10.1016/j.neuroscience.2007.11.026 (2008).

$71451 \quad$ Trappenberg, T. Fundamentals of Computational Neuroscience. 2nd edn, (Oxford

$715 \quad$ University Press, 2010).

71652 McCallum, A. \& Nigam, K. in AAAI-98 workshop on learning for text categorization

717 Vol. 752 (1998).

$71853 \quad$ Escabi, M. A. \& Schreiner, C. E. Nonlinear spectrotemporal sound analysis by

719 neurons in the auditory midbrain. J Neurosci 22, 4114-4131, doi:20026325

720 22/10/4114 [pii] (2002).

$72154 \quad$ Strong, S. P., de Ruyter van Steveninck, R. R., Bialek, W. \& Koberle, R. On the

722

723 application of information theory to neural spike trains. Pac Symp Biocomput, 621632 (1998). 
Fig1, Khatami; Escabi

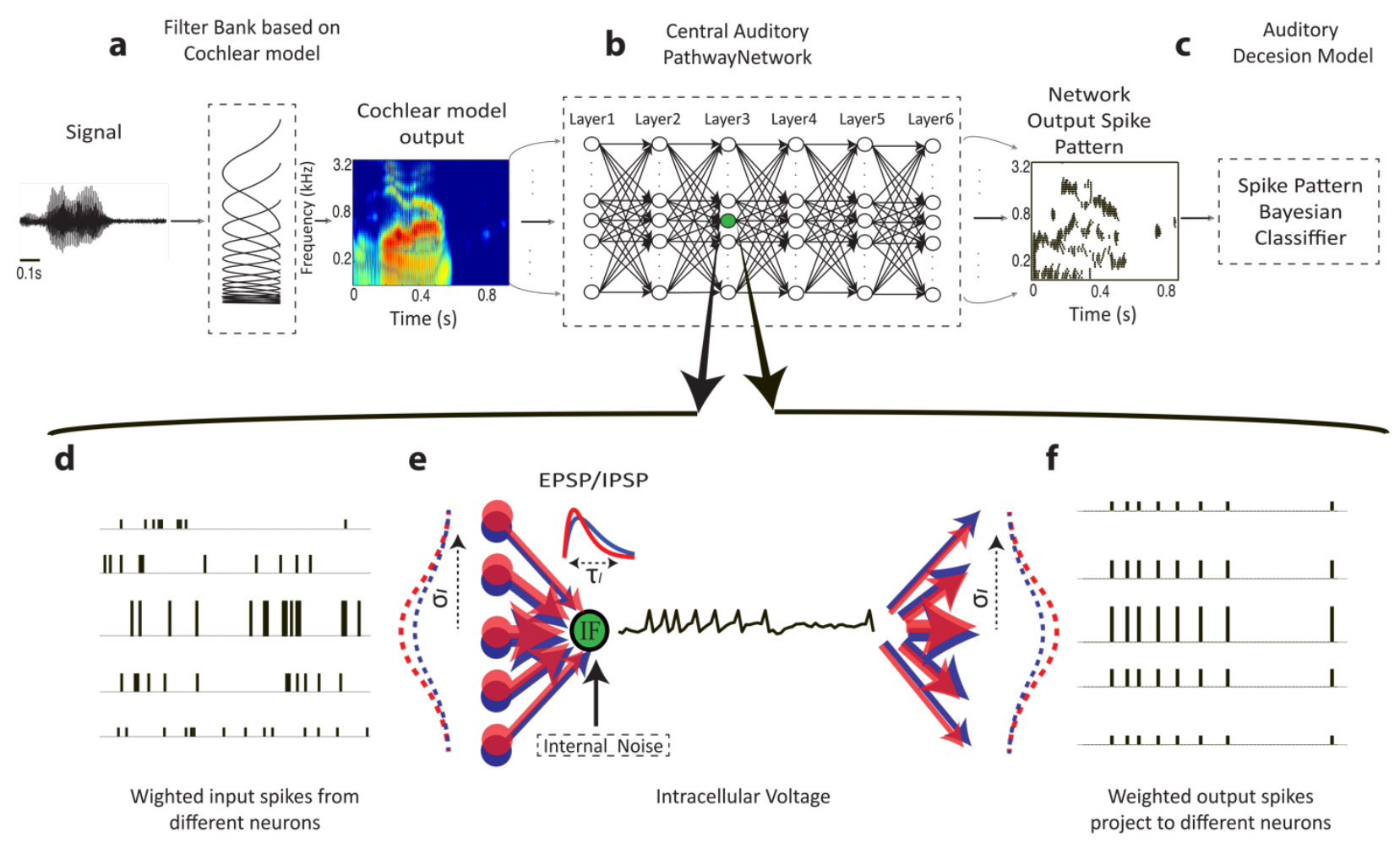

g
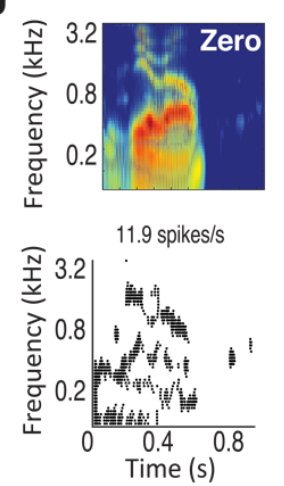
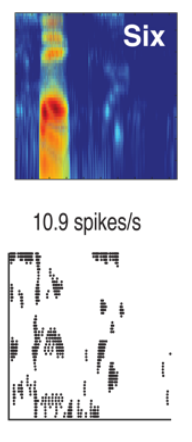

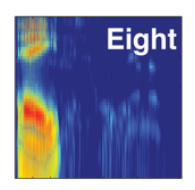

10.9 spikes/s

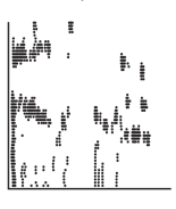

h
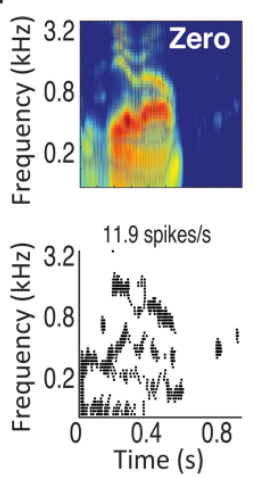
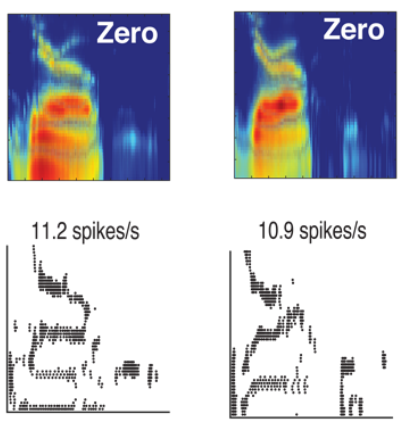

Figure 1. Auditory pathway hierarchical spiking neural network (HSNN) model. The model consists of a (a) cochlear model stage that transforms the sound waveform into a spectrogram (time vs. frequency), (b) a central hierarchical spiking neural network containing frequency organized spiking neurons and a (c) Bayesian classifier that is used to read the spatio-temporal spike train outputs of the HSNN. Each dot in the output represents a single spike at a particular time-frequency bin. (d-f) Zoomed in view of the HSNN illustrates the pattern of convergent and divergent connections between network layers for a single leaky integrate-and-fire (LIF) neuron. (d-e) Input spike trains from the preceding network layer are integrated with excitatory (red) and inhibitory (blue) connectivity weights that are spatially localized and model by Gaussian functions (f). The divergence and convergence between consecutive layers is controlled by the connectivity width (SD of the Gaussian model, $\sigma_{l}$ ). Each incoming spike generates excitatory and inhibitory postsynaptic potentials (EPSP and IPSP, red and blue kernels in $\mathbf{e}$ ). The integration time constant $\left(\tau_{l}\right)$ 
739 of the EPSP and IPSP kernels can be adjusted to control the temporal integration between 740 consecutive network layers while the spike threshold level $\left(N_{l}\right)$ is independently adjusted to control 741 the output firing rates and the overall neuron layer sensitivity. (g, h) Example cochlear model 742 outputs and the corresponding multi-neuron spike train outputs of the HSNN under the influence 743 of speech babble noise (at $20 \mathrm{~dB}$ SNR). (g) HSNN response pattern for one sample of the words 744 zero, six, and eight illustrate output pattern variability that can be used to differentiate words. (h) 745 Example response variability for the word zero from multiple talkers in the presence of speech 746 babble noise (20 dB SNR). 
Fig2, Khatami; Escabi

a
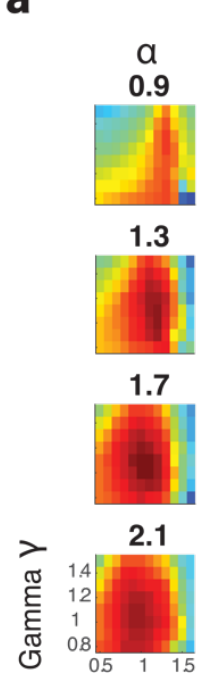

Lambda $\lambda$
$\mathrm{SNR}=5$

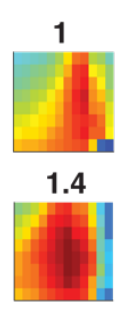

1.8

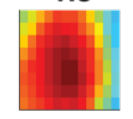

2.2

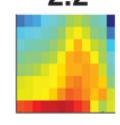

C
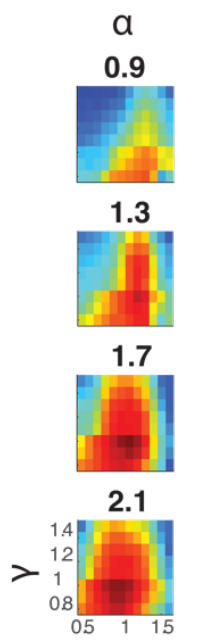

$\lambda$
2.3
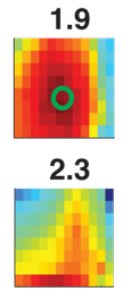

3

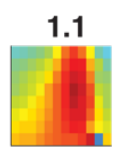

1.5

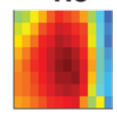

1.9

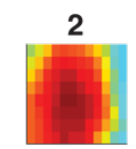

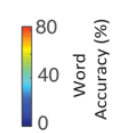
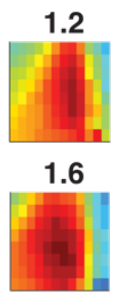

b

$a$
0.9

$S N R=20$

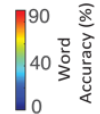

1.3

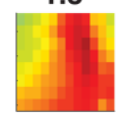

1.7
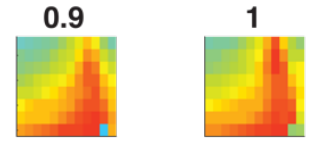

1.1

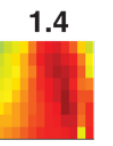

1.8
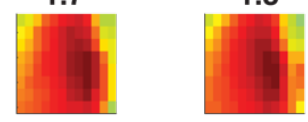

2.2
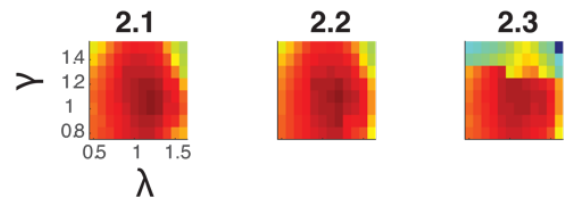

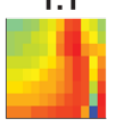

1.5

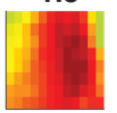

1.9
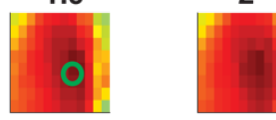

2

1.6
1.2
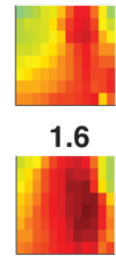

d

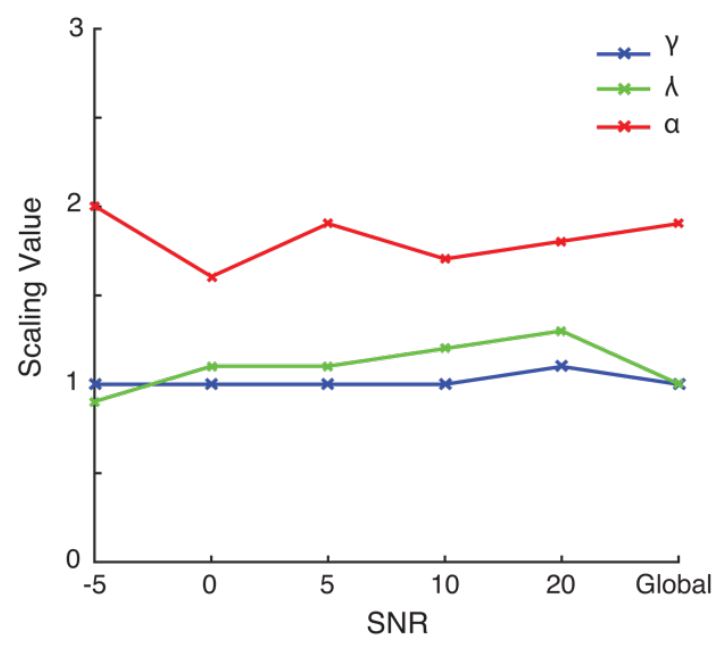

Figure 2. Hierarchical scaling is predicted by a global optimal solution that maximizes word recognition accuracy in the presence of background noise $(-5,0,5,10,15$ and $20 \mathrm{~dB}$ SNR). Crossvalidated word recognition accuracy (see Methods) is measured using the network outputs as a function of the three scaling parameters $(\alpha, \lambda$, and $\gamma)$. Word recognition accuracy curves are shown at 5 and $20 \mathrm{~dB}$ SNR (a and $\mathbf{b}$, respectively) as well as for the global solution (c, average accuracy between -5 and $20 \mathrm{~dB}$ SNR). In all cases shown, word recognition accuracy curves are tuned for the different scaling parameters and exhibit a similar optimal solution (green circles). (d) The optimal scaling parameters are relatively stable across SNRs and similar to the solution that maximize average performance across all SNRs (optimal solution $\alpha=1.9, \lambda=1.0$, and $\gamma=1.0$ ). 
Fig3, Khatami; Escabi

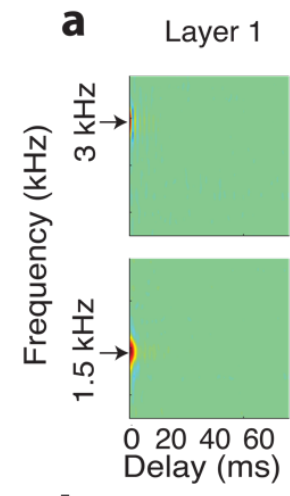

b
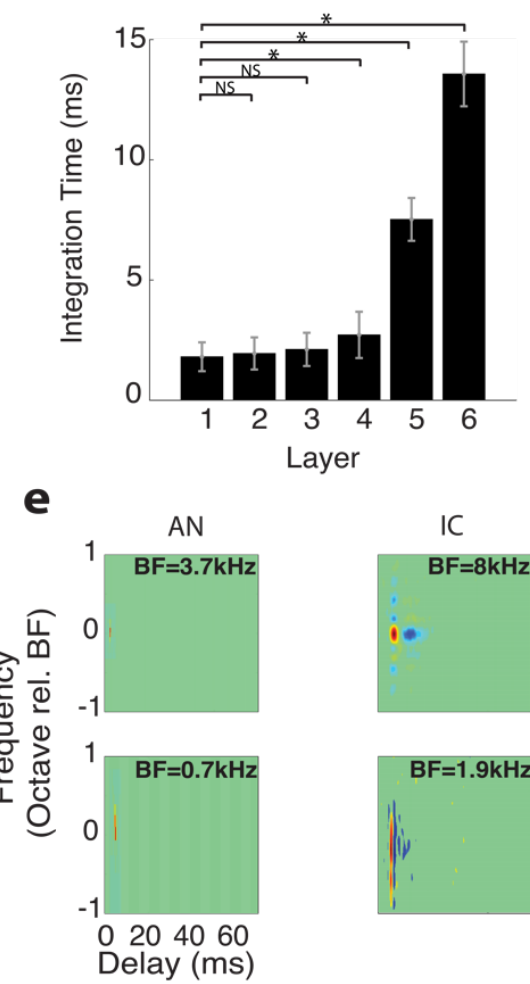

f
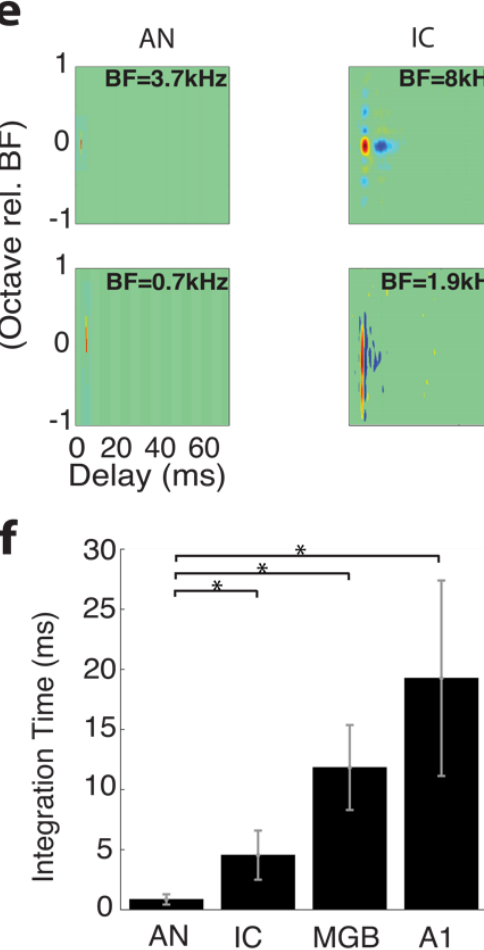

Layer 2

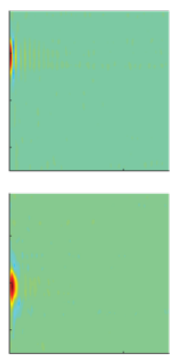

c

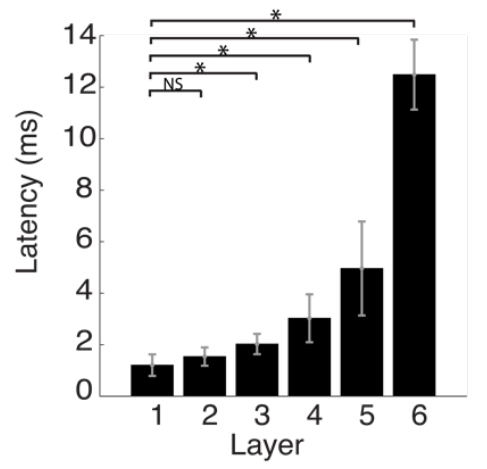

Layer 4
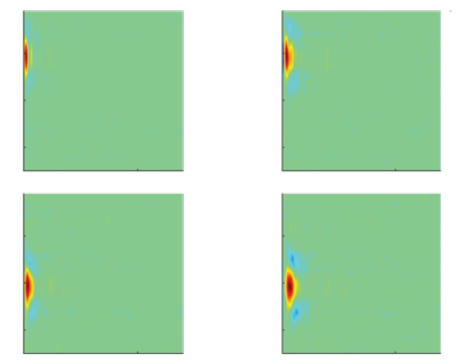

d

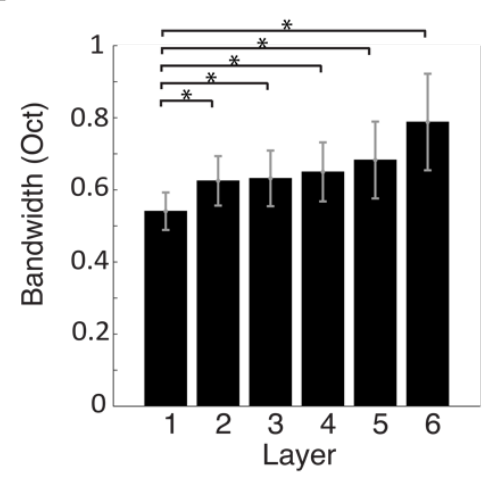

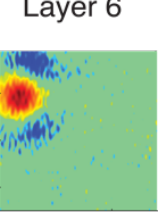
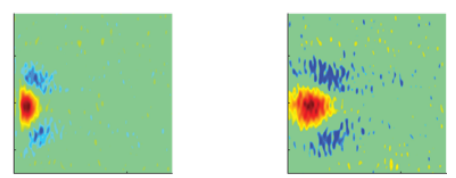

$1,1, k:$

Figure 3. Receptive field transformations of the optimal HSNN predicts transformations observed along the ascending auditory pathway. (a) Example spectro-temporal receptive field (STRF)

g

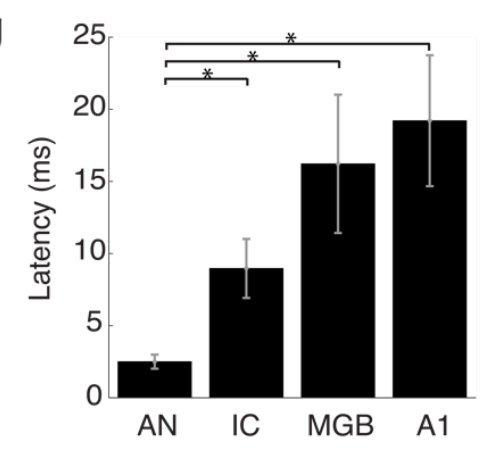

A1
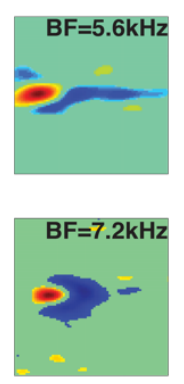

h

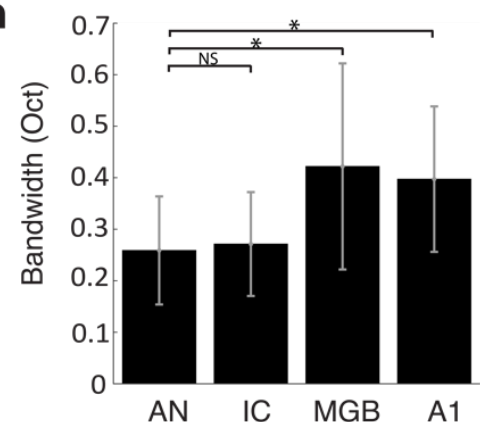


763 STRFs are normalized to the same color scale (red=increase in activity or excitation; 764 blue=decrease in activity or inhibition/suppression; green tones=lack of activity). In the early 765 network layers STRFs are relatively fast with short duration and latencies, and relatively narrowly 766 tuned. STRFs become progressively slower, slightly broader, and have longer and more varied 767 patterns of inhibition across the network layers, mirroring changes in spectral and temporal 768 selectivity observed in the ascending auditory pathway. The measured (b) integration times, (c) 769 latencies, and (d) bandwidths increase across the six network layers. (e) Examples STRFs from 770 the auditory nerve $(\mathrm{AN})^{26}$, inferior colliculus (IC) $)^{5}$, thalamus (MGB) and primary auditory cortex 771 (A1) $)^{6}$ become progressively longer and have progressively more complex spectro-temporal 772 sensitivity along the ascending auditory pathway. Average integration times (f), latencies (g) and 773 bandwidths (h) between AN and A1 follow similar trends as the optimal HSNN (b-d). Asterisks $774 \quad(*)$ designate significant comparisons (t-test with Bonferroni correction, $\mathrm{p}<0.01$ ) relative to layer 7751 for the optimal network (b-d) or relative to the auditory nerve for the neural data (f-h) while 776 error bars designate SD. 
Fig4, Khatami; Escabi

a
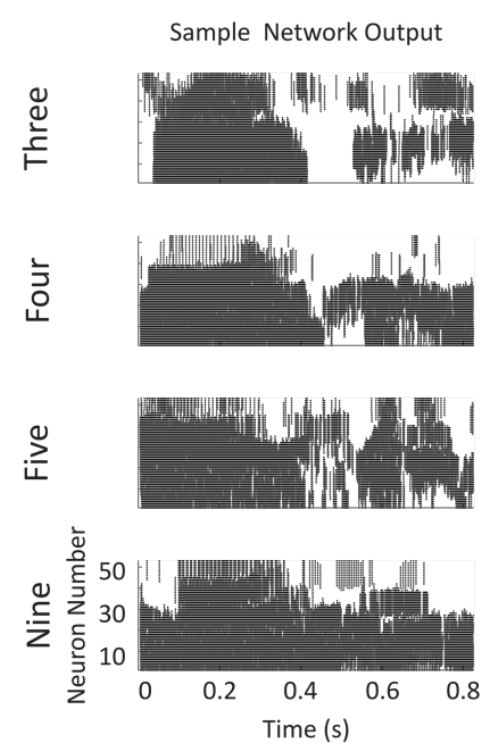

b

\section{Optimal HSNN}

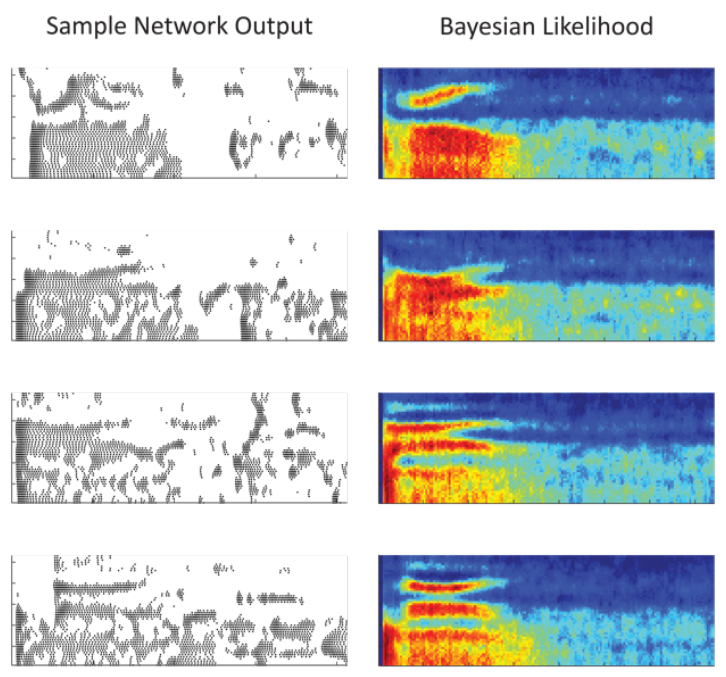

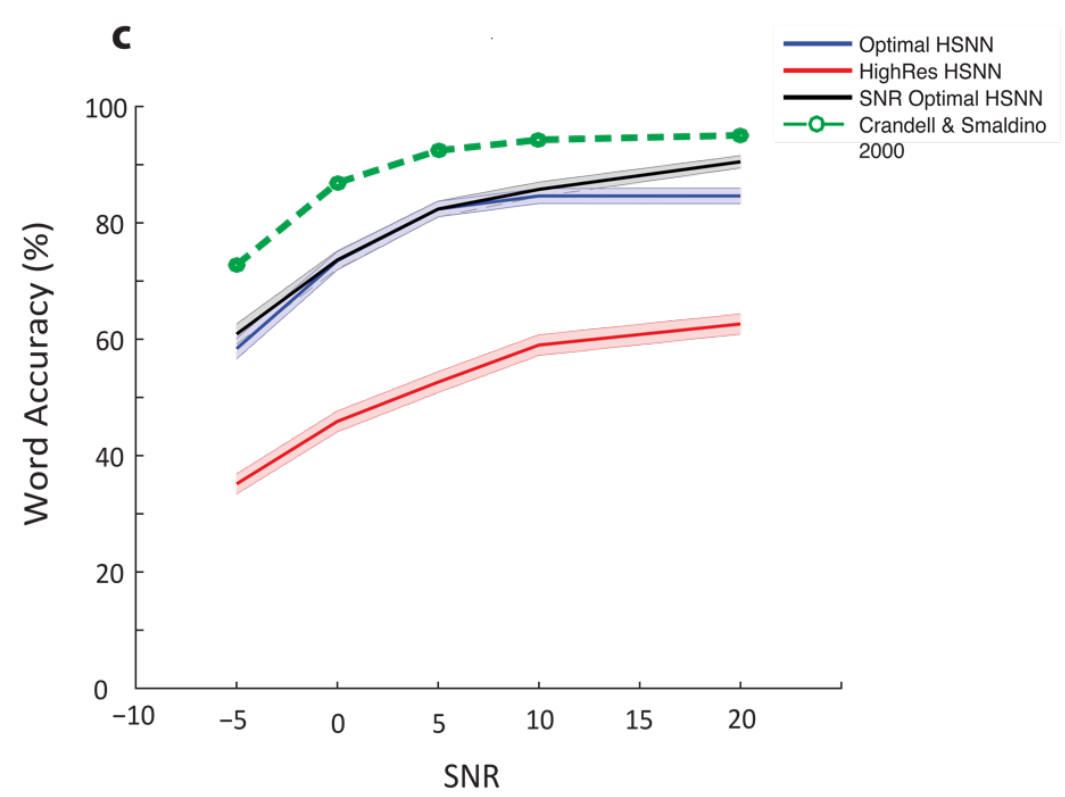

Figure 4. Optimal HSNN outperforms a high-resolution HSNN designed to preserve incoming acoustic information. Sample network spike train outputs and Bayesian likelihood histograms for the words three, four, five, and nine are shown for the (a) high-resolution and (b) optimal HSNN at $5 \mathrm{~dB}$ SNR. The Bayesian likelihood histograms correspond to the average probability of firing at each time-frequency bin for each digit (averaged across all trials and talkers). The firing patterns and Bayesian likelihood of the high-resolution network are spatio-temporally blurred compared to the hierarchical network. (b) Details such as spectral resonances (e.g., formants) and temporal transitions resulting from voicing onset are accentuated in the hierarchical network output. (c) The optimal HSNN (maximize performance across all SNRs) outperforms the high-resolution network in the word recognition task at all SNRs tested (blue=optimal; red=high-resolution) with an 
average accuracy improvement of $25.6 \%$. The optimal HSNN word recognition accuracy also closely matches the performance when the network is optimized and tested individually at each SNR (black, SNR optimal HSNN) indicative of a stable network representation. Finally, the optimal HSNN is within $\sim 10 \%$ of human performance in a similar word recognition task (dottedgreen curve ${ }^{32}$ ). 
Fig5, Khatami; Escabi
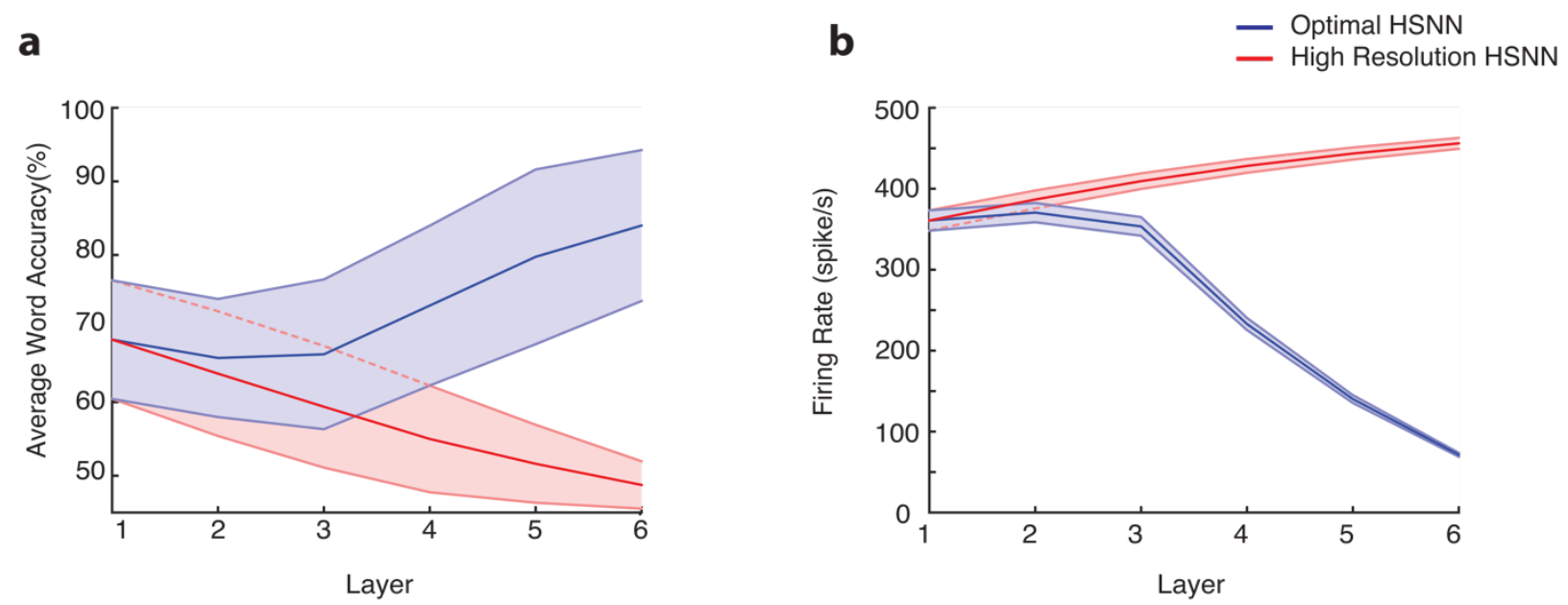

C
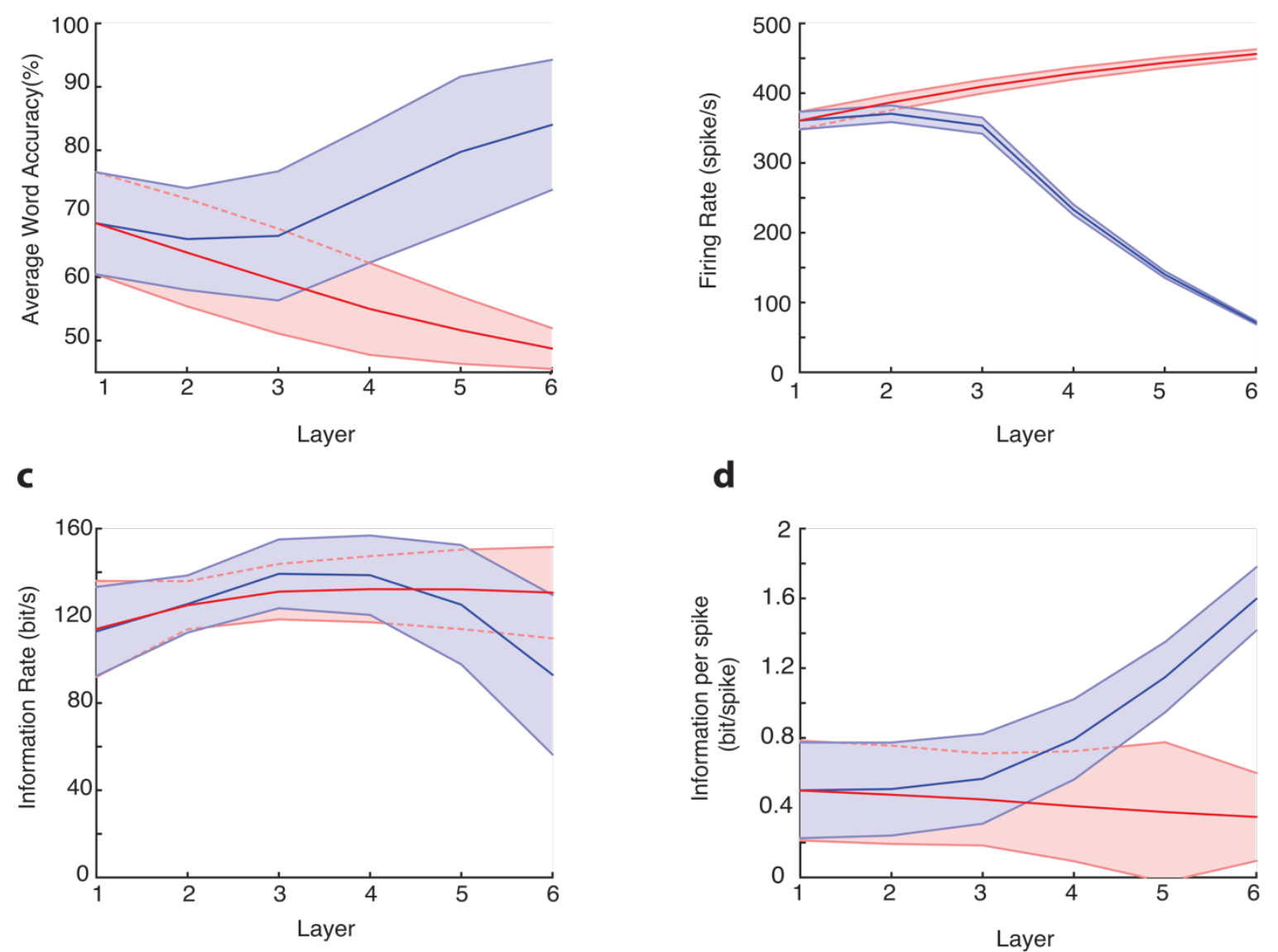

797

798

799

800

801

802

803

804

805

806

807

808

809

810

811

812

Figure 5. Hierarchical transformation between consecutive network layers enhances word recognition performance and robustness of the optimal HSNN. (a) The average word accuracy at $5 \mathrm{~dB}$ SNR systematically increases across network layers for the optimal HSNN (a, blue) whereas the high-resolution HSNN exhibits a systematic reduction in word recognition accuracy (a, red). For the high-resolution HSNN average firing rates (b, red), information rates (c, red), and information per spike (d, red) are relatively constant across layers indicating minimal transformations of the incoming acoustic information. In contrast, average firing rates $(\mathbf{b}$, blue) and information rates (c, blue) both decrease between the first and last network layers of the optimal network, consistent with a sequential sparsification of the response and a reduction in the acoustic information encoded in the output spike trains. However, the information conveyed by single action potentials (d, blue) in the optimal HSNN sequentially increase between the first and last layer so that individual action potentials become progressively more informative across layers. Continuous curves show the mean whereas error contours designate the SD. 
Fig6, Khatami; Escabi

a

STRFs

Models

Outputs

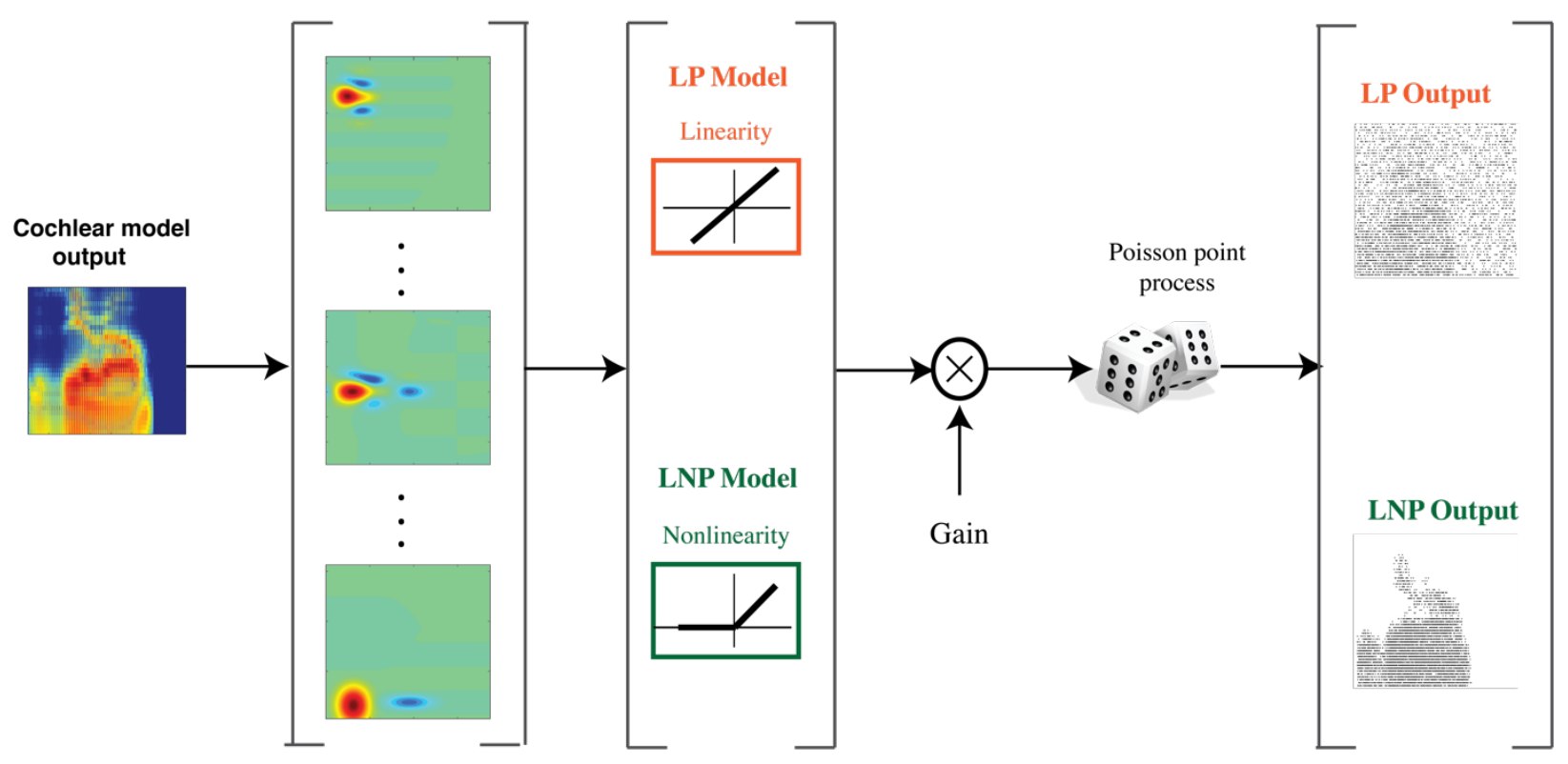

b

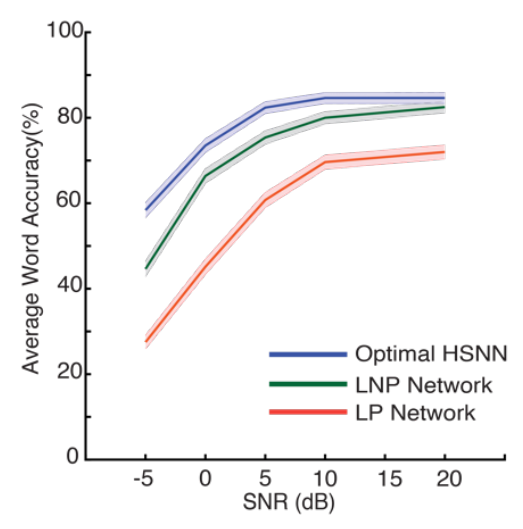

C

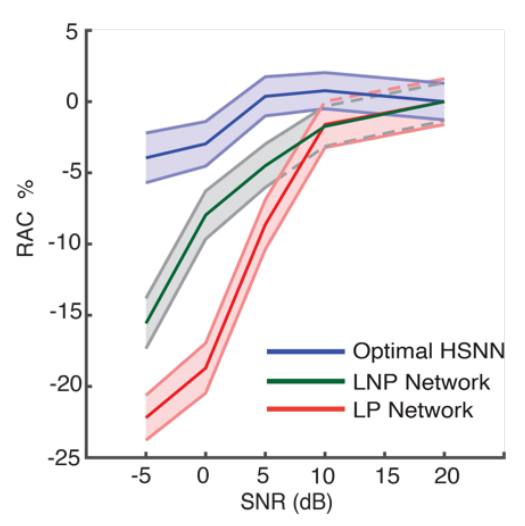

d

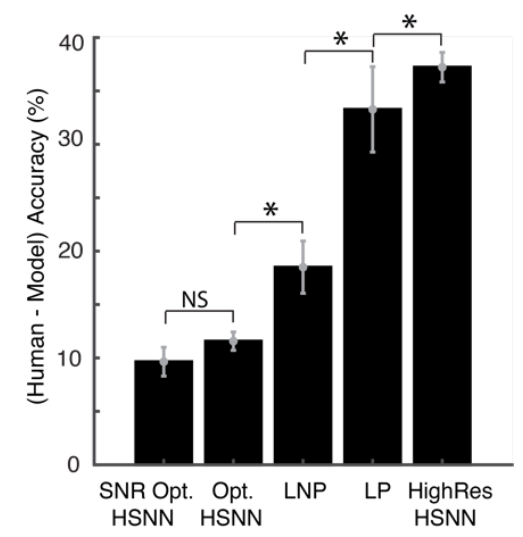

813

Figure 6. Optimal HSNN enhances robustness and outperforms single-layer generalized linear model networks with matched linear and nonlinear receptive field transformation. (a) Linear STRFs obtained at the output of the HSNN are used as to model the linear receptive field transformation of each neuron (see Methods). The LP network consists of an array of linear STRFs followed by a Poisson spike generator. The LNP network additionally incorporates a rectifying output stage following each STRF. (b) The optimal HSNN outperformance the LP network with an average performance improvement of $21.7 \%$ across SNRs. Nonlinear output rectification in the LNP network improves the performance to within $2 \%$ of the HSNN at $20 \mathrm{~dB}$ SNR. However, the average LNP performance was 7\% lower than the optimal HSNN and performance degraded systematically with increasing noise levels ( $13.75 \%$ performance reduction at $-5 \mathrm{~dB}$ SNR) demonstrating enhanced robustness of the optimal HSNN. (c) The relative accuracy change $\left(\mathrm{RAC}=\left(\mathrm{A}_{\text {model }}-\mathrm{A}_{\text {human }}\right)-\left(\mathrm{A}^{20 \mathrm{~dB}}{ }_{\text {model }}-\mathrm{A}^{20 \mathrm{~dB}}{ }_{\text {human }}\right)\right)$ was used to measure the divergence of each model across SNR when compared against human accuracy rates ${ }^{32}$. An RAC of 0 across SNRs indicates 
827 that the model performance follows a similar noise robust trend when compared to humans. For 828 the optimal HSNN, RACs were near zero across SNRs. RACs diverged substantially relative to 829 human accuracy rates with increasing SNR for the LP and LNP networks. (d) Average accuracy 830 difference between human and model data $\left(\mathrm{A}_{\text {human }}-\mathrm{A}_{\text {model }}\right)$. Average performance of the $\mathrm{SNR}$ 831 optimal (optimized for each SNR) and optimal HSNN (optimized across all SNRs) are within 10 $832 \%$ of the human word accuracy rates. The LNP (18.5\%), LP (33.3\%) and high-resolution HSNN $833(37.2 \%)$ performance are substantially lower relative to humans. Asterisks designate significant 834 differences $(\mathrm{p}<0.05$, $\mathrm{t}$-test with Bonferroni correction) and error bars designate SEM.

835

836 
Fig7, Khatami; Escabi
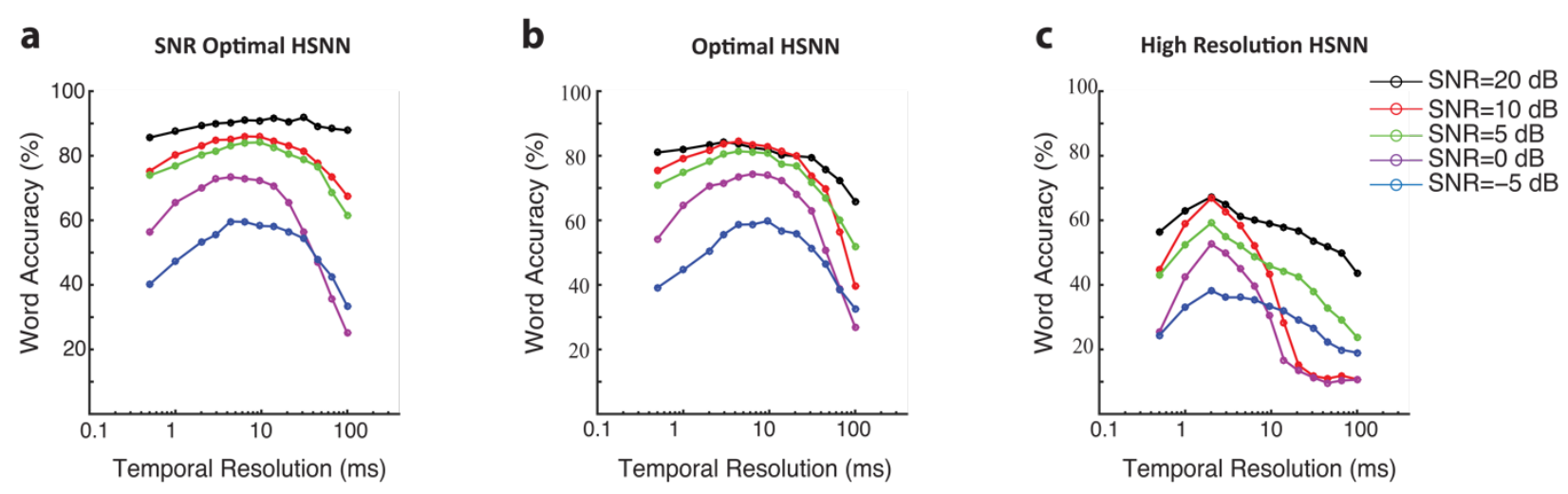

Figure 7. Optimal temporal resolution that maximize word recognition accuracy in noise. (a) Word accuracy rate as a function of spike train temporal resolution (bin widths 0.5-100 mms) and SNR $(-5$ to $20 \mathrm{~dB})$ for the optimal (a) and high resolution networks (c). Each curve is computed by selecting the optimal scaling parameters for each SNR and measuring the word accuracy rate from the network outputs at multiple temporal resolutions. (b) Same as (a), except that global optimal scaling parameters were used for all SNRs tested. The temporal resolution that maximizes the word accuracy rate of the global optimal HSNN is $6.5 \mathrm{~ms}$. (c) Word accuracy rate as a function of temporal resolution and SNR for the high-resolution network. The optimal temporal resolution for the high-resolution HSNN is $2 \mathrm{~ms}$. 
Fig1S, Khatami; Escabi

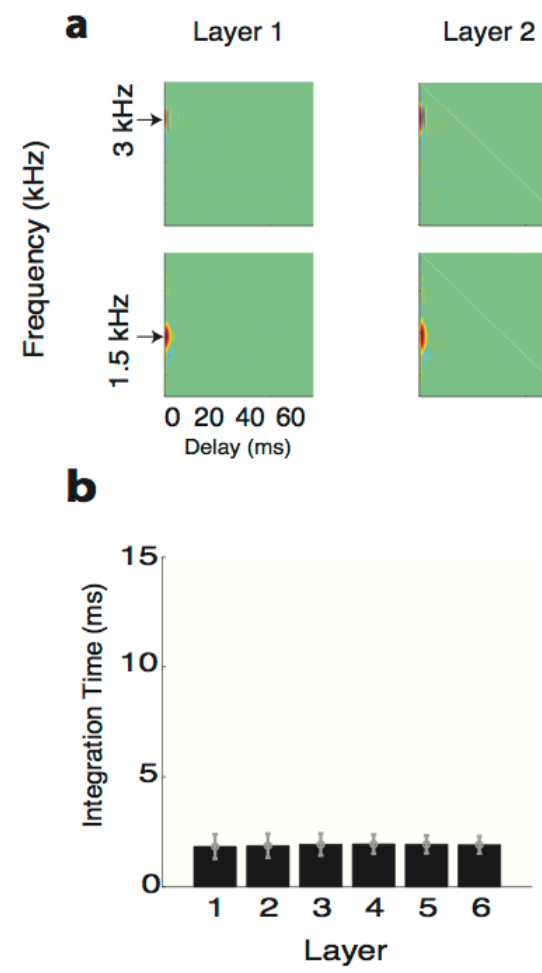

C

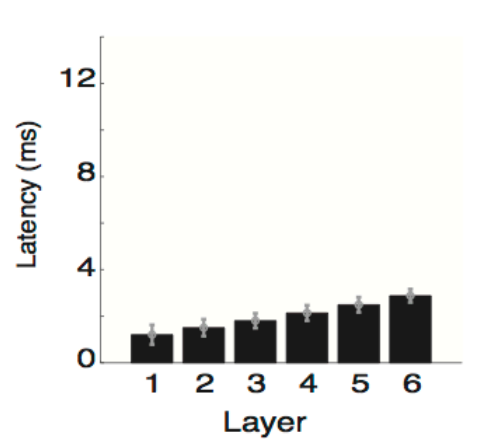

Layer 4
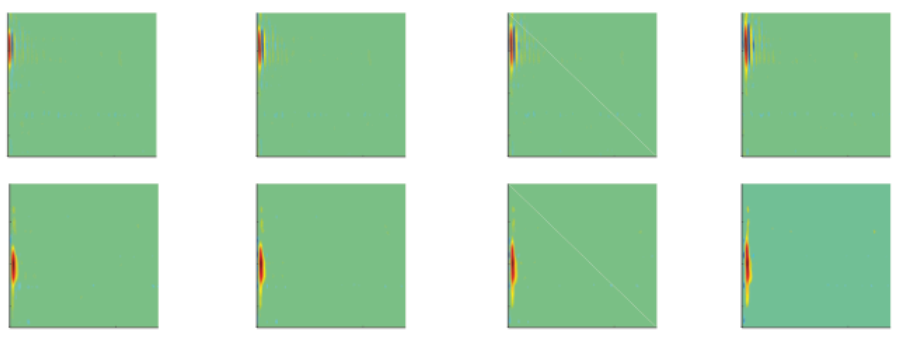

d

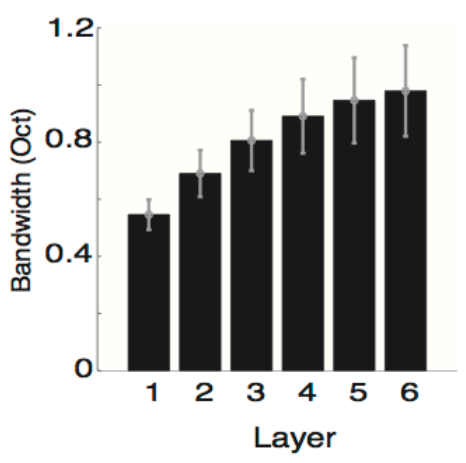

Figure 1S. Receptive field transformations of the high-resolution network indicate that spectrotemporal information propagates with minimal processing across network layers. (a) Example spectro-temporal receptive field (STRF) measured for the optimal network maintain highresolution and change minimally across network layers. Unlike the optimal network, the measured (b) integration times and (c) latencies change minimally and are relatively constant across the six network layers. (d) Bandwidths, by comparison, increase slightly across the six network layers and follow a similar trend as the optimal HSNN. The figure format follows the same convention as in Figure 3. 